\title{
DETERMINANTES DE LA INNOVACIÓN Y LA PRODUCTIVIDAD EN LAS SUBSIDIARIAS EXTRANJERAS Y LAS EMPRESAS EXPORTADORAS EN LA INDUSTRIA EN COLOMBIA
}

\section{DETERMINANTS OF INNOVATION AND PRODUCTIVITY IN FOREIGN SUBSIDIARIES AND EXPORTING FIRMS IN THE COLOMBIAN INDUSTRY}

Kruscalla Albis Salas, Nadia (Observatorio Colombiano de Ciencia y Tecnología) *

\section{RESUMEN}

En esta investigación se evalúan los determinantes de la innovación y su efecto sobre la productividad en las empresas extranjeras y domésticas exportadoras utilizando datos de sección cruzada a nivel de empresa para la industria manufacturera en Colombia. Entre los principales hallazgos están: (i) el esfuerzo realizado por las empresas exportadoras y las subsidiarias extranjeras en actividades de innovación favorece la obtención de innovaciones tanto en productos como en procesos. Sin embargo difieren tanto en el nivel de los rendimientos de la inversión obtenidos por la inversión en innovación como en el tipo de actividad innovadora en que se basa la producción de innovaciones; (ii) tan sólo la innovación en producto tiene un impacto positivo en la productividad, en especial cuando la producción de innovaciones está relacionada con la ejecución de actividades de I+D y (iii) entre las principales variables con efectos relevantes sobre la innovación en las empresas internacionalizadas están las barreras financieras, la disponibilidad de recursos humanos, la protección de innovaciones, la cooperación, en especial, en la cadena de producción, y en el caso específico de las subsidiarias su posición en el grupo multinacional.

Palabras claves: Innovación, productividad, internacionalización, subsidiarias extranjeras, empresas exportadoras. JEL: O31, O32, O38.

\begin{abstract}
This paper investigates the determinants of innovation and productivity in foreign subsidiaries and national exporters firms, using cross-sectional data at firm level for the industry in Colombia. The major findings suggest that: (i) the effort made by export companies and foreign subsidiaries in innovation activities favours the obtaining of innovations in both product and process. However, they differ in the level of investment returns and the type of innovation activities in which is based the knowledge production; (ii) only product innovation has a positive impact on productivity, especially when it is the result of the implementation of R\&D activities and (iii) the main factors that affect innovation in exporting companies and foreign subsidiaries are financial barriers, levels of human resources, protection of innovations, cooperation with clients and suppliers and, specifically, in foreign subsidiaries their position in the multinational group.
\end{abstract}

Key words: Innovation, productivity, internationalization, foreign subsidiaries, exporting companies. JEL: O31, O32, O38.

Observatorio Colombiano de Ciencia y Tecnología, Carrera 15 \#37-59, Bogotá D.C., Colombia. nkruscalla@gmail.com

Recibido: Septiembre de 2015. Aceptado: Octubre de 2015. 


\section{INTRODUCCIÓN}

En la literatura teórica y empírica reciente se ha documentado que la heterogeneidad tecnológica y productiva de las empresas está influenciada por el grado de internacionalización de sus actividades ${ }^{1}$. La evidencia indica que las multinacionales muestran un nivel de productividad mayor que las empresas exportadoras y éstas, a su vez, superan a las empresas domésticas sin presencia en los mercados internacionales (Aitken y Harrison, 1999; Doms y Jensen, 1998; Girma et al., 2005; Griffiths et al., 2002). Asimismo, se ha constatado que las empresas internacionalizadas mantienen un mayor desempeño innovador que las empresas no conectadas a los mercados externos (Castellani y Zanfei, 2007; Criscuolo et al., 2010; Frenz y Ietto-Gillies, 2007; Siedschlag et al., 2010).

Dada la heterogeneidad establecida por el grado de conexión con los mercados externos, cabría esperar que varíen los determinantes, así como el nivel de sus efectos, de la capacidad innovadora y productiva de las empresas extranjeras, de las firmas domésticas exportadoras y domésticas no exportadoras, al ser tomadas como unidades específicas de análisis ${ }^{2}$. De acuerdo con la revisión de la literatura, este tipo de aproximación ha sido más frecuente en el caso de las subsidiarias de empresas extranjeras y en menor medida para las firmas exportadoras. Por ejemplo, Cantwell y Mudambi (2005) analizan los determinantes de la intensidad del gasto en Investigación y Desarrollo (I+D) entre las subsidiarias extranjeras ubicadas en el Reino Unido, mientras que Álvarez y Cantwell (2011) analizan la innovación y los factores que explican los diferenciales de productividad entre subsidiarias foráneas que residen en España.

Teniendo en cuenta lo anterior, el objetivo de la presente investigación consiste en identificar los determinantes de la innovación y la productividad en las empresas internacionalizadas (domésticas exportadoras y subsidiarias de empresas extranjeras) en comparación con las empresas no conectadas a los mercados globales (domésticas no exportadoras). Se utilizan para este estudio los datos de la quinta versión de la Encuesta de Desarrollo e Innovación Tecnológica -EDIT V- realizada por el Departamento Administrativo Nacional de Estadística de Colombia (DANE) y sus correspondientes variables de comportamiento económico derivadas de la Encuesta Anual Manufacturera EAM- elaborada por la misma entidad.

El documento se estructura de la siguiente manera. En la segunda sección se presenta el marco conceptual que soporta la investigación; en la tercera, se desarrolla la estrategia econométrica aplicada, y en la cuarta sección se muestran los datos y las estadísticas descriptivas. Los resultados de las estimaciones se presentan en la quinta sección y, finalmente, en la sexta sección se plantean las conclusiones.

\footnotetext{
${ }^{1}$ La internacionalización puede definirse como la expansión de las firmas hacia ubicaciones geográficas fuera de las fronteras nacionales (Hitt et al., 1997), a través de dos vías básicas: la exportación o la inversión extranjera directa.

${ }^{2}$ Es decir, utilizar muestras truncadas para cada tipo de empresa: subsidiarias extranjeras, empresas domésticas exportadoras y empresas domésticas no exportadoras.
} 


\section{MARCO CONCEPTUAL}

Existen varios factores que afectan a la innovación y a la productividad dentro de las firmas internacionalizadas. A continuación se presentan ciertos rasgos específicos señalados en la literatura para las empresas multinacionales y las exportadoras, para posteriormente abordar aquellos que constituyen rasgos comunes a cualquier tipo de empresa y sobre los que se espera evaluar su impacto diferenciado sobre la innovación y la productividad.

\section{a. Innovación, productividad y subsidiarias extranjeras ${ }^{3}$}

El desempeño tecnológico y productivo difiere entre diferentes tipos de subsidiarias dependiendo de múltiples factores tanto internos como externos a las mismas. Entre los más importantes se pueden señalar: (i) el tipo de mandato asignado a las subsidiarias que define su posición en el grupo multinacional (Cantwell y Mudambi, 2005) (ii) la estructura de propiedad y las formas de entrada de la inversión extranjera, ya sea por medio de inversiones greenfield o fusiones y adquisiciones (Almeida y Fernandes, 2008; Álvarez y Marín, 2010); (iii) la nacionalidad de las subsidiarias y, en particular, los efectos de diferentes estilos de gobernanza corporativa (Dachs et al., 2008); (iv) las relaciones que se establecen con actores locales que se sustentan en la disponibilidad de capital humano y una infraestructura en investigación de la economía receptora (Cantwell y Molero, 2003), y (v) el sector industrial en el que se encuentran las subsidiarias, pues estos definen diferentes oportunidades tecnológicas (Balcet y Evangelista, 2005). No todos estos factores pueden ser medidos con la información disponible, de ahí que en la presente investigación nos centramos en dos de ellos: (i) la posición de la subsidiaria en el grupo multinacional y (ii) los vínculos con organizaciones del sistema de innovación de la economía receptora.

En primer lugar, una de las fuentes de heterogeneidad en el desempeño innovador y productivo de las subsidiarias tiene que ver con los mandatos asignados a las mismas. En sentido amplio, la noción de mandato se relaciona con las responsabilidades asignadas a las subsidiarias en los negocios y actividades que se extienden más allá de los mercados nacionales de origen y de destino, y que definen su forma de especialización en la red que conforma el grupo multinacional (Birkinshaw y Hood, 1998). Esta especialización define la posición de una subsidiaria en la cadena de valor y también en las actividades de innovación de la corporación.

Desde el punto de vista tecnológico, con efectos sobre lo productivo, dos tipos de mandatos se pueden distinguir (Cantwell y Mudambi, 2005): (i) las subsidiarias explotadoras de las competencias que ya posee la empresa multinacional o competence exploiting y (ii) las subsidiarias creadoras de competencias o competence creating. En el primer caso, las actividades de innovación generalmente se dirigen hacia la adaptación de los productos y procesos a las condiciones de los mercados locales. En el segundo tipo de subsidiarias, las estrategias tecnológicas buscan la creación o adquisición de competencias tecnológicas nuevas o complementarias, que aumenten su stock de conocimiento y mejoren la capacidad de la corporación multinacional para obtener innovaciones tanto para los mercados locales como globales.

Entre los elementos que permiten aproximarse a la evolución de las subsidiarias hacia un mandato de creación de competencias está su integración en los mercados internacionales

\footnotetext{
${ }^{3}$ Se utiliza el término de subsidiarias extranjeras para diferenciarlas de las subsidiarias de empresas nacionales o de las subsidiarias de empresas nacionales que operan en el extranjero.
} 
y en la corporación multinacional. Aunque las subsidiarias pueden iniciar sus operaciones como unidades que sirven a los mercados domésticos del país de acogida, gradualmente pueden orientarse hacia la exportación e integrarse más en las operaciones internacionales, ya sea tecnológicas o productivas, de su grupo multinacional (Álvarez y Cantwell, 2011; Cantwell y Mudambi, 2005). La mayor conexión de las subsidiarias con los mercados externos amplía los canales de aprendizaje de las mismas, lo cual puede derivar en que se conviertan en unidades de la empresa con actividades tecnológicas más avanzadas aunque no necesariamente en la frontera tecnológica. Por su parte, la mayor integración de las subsidiarias en su grupo multinacional permite que exista un mayor potencial innovador debido a que cada unidad del grupo aprende del entorno en que opera y transmite el conocimiento al interior de la empresa, beneficiándose de la transferencia de conocimiento y del aprendizaje logrado en sus entornos específicos (Frenz y Ietto-Gillies, 2007). Esta dinámica puede dar lugar a la existencia de flujos de conocimiento tanto convencionales (desde la casa matriz a la filial) como reversos (de la filial a la casa matriz) que tienen importantes implicaciones sobre los procesos de aprendizaje en las multinacionales.

De lo anterior se espera, entonces, que las empresas que exportan y mantienen una posición tecnológica de alguna relevancia en su grupo multinacional, ya sea hacia la adaptación o a la creación de nuevos productos, muestren un desempeño tecnológico superior, lo que a su vez se reflejaría en ganancias en productividad. Adicionalmente, relacionado también con el concepto de mandato, son relevantes los vínculos con organizaciones del país de recepción de la inversión extranjera. En especial, en el caso de las subsidiarias con un mandato de creación de competencias, resulta de gran relevancia la disponibilidad de habilidades locales, capital humano y una infraestructura que les permitan profundizar y ampliar su conocimiento tecnológico a través de su inserción en las redes locales (Cantwell y Molero, 2003). De hecho, se ha encontrado que las empresas altamente vinculadas con el entorno local muestran un fuerte compromiso con las actividades de innovación, una alta propensión a introducir innovaciones de producto y un alto nivel de recursos dedicados a la I+D (Balcet y Evangelista, 2005). Por lo tanto, se espera que las relaciones de cooperación de las subsidiarias con agentes locales sea un determinante importante de la innovación y, por ende, de la productividad.

\section{b. Innovación, productividad y exportación}

En los estudios sobre la relación entre innovación, productividad y exportaciones se pueden distinguir dos corrientes. La primera de ellas plantea que la innovación es la que determina la actividad exportadora, basándose en la hipótesis según la cual las firmas más productivas y más innovadoras se autoseleccionan en los mercados internacionales (Helpman et al., 2004) ${ }^{4}$. El segundo tipo de literatura propone la relación inversa, es decir, que la actividad exportadora determina las capacidades de innovación de las empresas; este caso nos remite a la hipótesis del aprendizaje mediante la exportación o learning by exporting (Clerides et al., 1998). El presente trabajo se circunscribe en mayor medida en esta última corriente, pero añade un componente adicional al análisis consistente en evaluar bajo qué

\footnotetext{
${ }^{4}$ Cuando la empresa innova, consigue mejorar su posición competitiva en los mercados internacionales y, en consecuencia, aumenta su propensión a exportar y a vender una mayor fracción de su producción en esos mercados (Cassiman et al., 2010). Al mejorar la calidad de los productos (i.e innovación de producto) las empresas consiguen desplazar la curva de demanda de exportaciones y así aumentar sus ventas en el exterior.
} 
condiciones o determinantes las empresas exportadoras son más innovadoras y más productivas.

Es amplia la literatura que ha encontrado evidencia en relación a que las empresas aprenden en su proceso de internacionalización y, por tanto, que la decisión de iniciar la actividad exportadora tiene importantes consecuencias sobre la innovación de la empresa (Aw et al., 2005; Bratti y Felice, 2012). En particular, se argumenta que la actividad exportadora implica para la empresa tener acceso a la tecnología desarrollada en el exterior que no está disponible en el mercado doméstico, a la que se puede acceder, por ejemplo, a través del contacto con intermediarios o clientes externos. A su vez, este conocimiento adquirido puede posteriormente incorporarse a la actividad productiva local, llevando así a las empresas que exportan a innovar o a hacerlo con mayor intensidad.

Con la exposición en los mercados internacionales las firmas exportadoras obtienen varias ventajas en términos del aprendizaje, entre las que se pueden señalar (Salomon y Shaver, 2005): (1) beneficiarse de la experiencia y habilidades de sus compradores y recibir información valiosa acerca de las preferencias de los consumidores con respecto a los productos con los que compiten; (2) posibilidad de recibir apoyo de sus proveedores en el diseño de productos y asistencia técnica para mejorar tecnologías de proceso y (3) la posibilidad de acceder a las mejores prácticas disponibles.

De acuerdo con lo expuesto, es de esperar que las empresas que han logrado acceder a los mercados internacionales y acumular experiencia muestren un desempeño innovador y productivo superior. De igual forma que en el caso de las subsidiarias, no todas las empresas que exportan son innovadoras o en el caso en que lo sean no necesariamente obtienen innovaciones con alto grado de novedad o de tipo radical. Esto sugiere que es necesario identificar factores adicionales que influyen en la capacidad de las empresas exportadoras para realizar un mayor esfuerzo innovador, producir innovaciones y obtener ganancias de productividad. A continuación se presentan algunos determinantes de la innovación que pueden ser evaluados tanto para las exportadoras como para las subsidiarias de empresas multinacionales.

\section{c. Determinantes de la innovación y la productividad}

Ciertos determinantes de la innovación o la productividad suelen ser comunes a todas las empresas independientemente de la heterogeneidad empresarial observada (HurmerintaPeltomäki, 1996). A este respecto, el presente estudio incluye aquellos factores que se han señalado en la literatura que evalúan la relación entre innovación y productividad.

Un primer factor a tener en cuenta es el tamaño empresarial. Al respecto, existen argumentos que sustentan la hipótesis de que las grandes empresas son más innovadoras que el resto de firmas debido a tres factores principales (Cohen, 2010): (i) mayor capacidad de acceder a financiación externa y mayor disponibilidad de recursos propios; (ii) mayor diversificación de productos para enfrentar la incertidumbre de la innovación y (iii) existencia de economías de escala en la función de $\mathrm{I}+\mathrm{D}$, economías de alcance por la complementariedad entre la I+D y otras actividades corporativas (por ejemplo el marketing). Pero por el contrario, hay otros argumentos que sostienen que las pequeñas pueden ser más innovadoras en contextos de alta dinámica tecnológica, debido a su mayor flexibilidad para adaptarse a cambios en el entorno (Cohen y Klepper, 1996).

Un segundo factor, es el poder de mercado que poseen las empresas como determinante de las condiciones que dan lugar a la innovación. Las presiones competitivas pueden generar incentivos para innovar y, de esa manera, las empresas pueden lograr elevar su participación 
en el mercado en el futuro, de manera similar al efecto del crecimiento del tamaño empresarial. El incremento del poder de mercado genera medios financieros para innovar y reduce los niveles de riesgo del proceso innovador (Bozeman y Link, 1983).

Un tercer factor incluido en el análisis es la cualificación del recurso humano. Los procesos de innovación en las firmas dependen sustancialmente de su capital de conocimiento, el cual consiste en todas las habilidades y el conocimiento que la firma posee para soportar la innovación (Klette y Kortum, 2002). Las empresas dependen de sus competencias internas, entre ellas sus recursos humanos para evaluar, seleccionar y adoptar tecnologías potencialmente disponibles de otros agentes, así como para desarrollar internamente innovaciones dentro de su estructura organizativa (Cohen y Levinthal, 1990). El tipo de capacidades relacionadas con las habilidades del recurso humano no siempre están conectadas a procesos de innovación formales en unidades de I+D establecidas, sino que más bien se encuentran distribuidas en las organizaciones en las que se desenvuelven procesos de learning by doing (Arrow, 1962).

Un cuarto factor son las condiciones de apropiación del conocimiento ${ }^{5}$. En la literatura se han planteado dos posibles efectos de la existencia de bajos niveles de apropiación de la innovación (Cohen, 2010): (i) desincentivo a la realización de actividades de innovación por la incapacidad de las empresas de apropiarse de sus beneficios; y (ii) el efecto contrario, los bajos niveles de apropiación permiten altos niveles de flujos de conocimiento o spillovers entre organizaciones de la economía. Los mecanismos de los que disponen las empresas para proteger sus innovaciones pueden ser formales, como las patentes, o informales, como el secreto industrial, la complejidad en el diseño, los acuerdos de confidencialidad o la cooperación con competidores. En el caso de las subsidiarias, la protección formal de innovaciones, como las patentes, puede ser especialmente relevante debido a la alta propensión a patentar de las multinacionales y también a la necesidad de proteger las innovaciones ya existentes en el grupo multinacional (Criscuolo et al., 2010).

El sector donde se ubican las empresas es el quinto factor considerado al evaluar la innovación y la productividad. Los sectores difieren sustancialmente debido a la existencia de características particulares definidas por las oportunidades tecnológicas, las bases de conocimiento, las condiciones de apropiación de la innovación y la acumulación del avance técnico (Dosi, 1988; Malerba, 2002; Nelson y Winter, 1982; Pavitt, 1984). En primer lugar, no en todos los sectores se tienen las mismas oportunidades tecnológicas, es decir, el rendimiento de la inversión en $\mathrm{I}+\mathrm{D}$ no es el mismo en términos del desarrollo de innovaciones. En segundo lugar, los sectores difieren en la capacidad de acumular el conocimiento generado y de aprovecharlo para producir mayor conocimiento en el futuro. Adicionalmente, las condiciones para proteger la innovación de la imitación y capturar los beneficios de sus innovaciones son distintas entre sectores. Por último, difieren también las bases de conocimiento que sustentan las actividades de innovación de las firmas y su naturaleza ya sea de carácter tácito o codificado, simple o complejo, genérico o especializado.

Un sexto factor incluido en el estudio es la interacción de las empresas con otras organizaciones. Las firmas se enfrentan a un conjunto de opciones para llevar a cabo sus actividades de innovación (Bayona et al., 2001): 1) su ejecución dentro de la propia organización; 2) la adquisición externa de recursos de conocimiento o, 3) a través de la

\footnotetext{
${ }^{5}$ Estas se refieren a la posibilidad que tienen los innovadores de proteger la innovación de la imitación y capturar los beneficios de sus innovaciones. Justamente, una característica esencial del proceso innovador es la baja capacidad para apropiarse del conocimiento producido, pues su uso y explotación no está limitado sólo a quienes realizan actividades innovadoras.
} 
cooperación con otras entidades. Los motivos y las ventajas de la cooperación en innovación varían también con el tipo de socios (Belderbos et al., 2004; Tether, 2002). Mediante la cooperación con clientes la empresa adquiere un mejor conocimiento de las necesidades que estos presentan y, por tanto, se reduce el riesgo asociado con llevar la innovación al mercado; mientras que la cooperación con proveedores permite minimizar costes de transacción, aparte de generar aprendizaje conjunto y confianza. La cooperación con competidores permite establecer estándares en la introducción de nuevos bienes o servicios y complementariedad en el desarrollo de innovaciones cuando los socios poseen competencias diferentes que son costosas de adquirir. Asimismo, la cooperación con organizaciones de $\mathrm{I}+\mathrm{D}$, tales como universidades y centros de investigación públicos, les permite a las empresas acceder a nuevo conocimiento de manera rápida, poco costosa y privilegiada, lo que complementa los esfuerzos internos en $\mathrm{I}+\mathrm{D}$, al proporcionarles a las empresas una ventana a tecnologías emergentes y también acceder a un soporte tecnológico especializado (por ejemplo, expertos y especialistas). Además de la cooperación y las transacciones de mercado, el conocimiento externo se puede adquirir a través del aprovechamiento de los denominados spillovers, que son los flujos de ideas que surgen del stock de conocimiento existente (Griliches, 1979). Sin embargo, las empresas no pueden beneficiarse de este stock si no tienen las capacidades de absorción necesarias, pues éstas determinan su habilidad para identificar, asimilar y explotar el conocimiento generado externamente (Cohen y Levinthal, 1990). Por otra parte, la capacidad de absorber conocimiento externo depende asimismo de las condiciones de apropiación del conocimiento.

Otro factor central para fortalecer la capacidad y el éxito innovador de las empresas es la financiación pública de las actividades de innovación. Su justificación se apoya en el argumento de que los fallos de mercado, tales como información asimétrica o problemas de apropiación del conocimiento derivan en un nivel sub-óptimo de inversión privada en actividades innovadoras (Arrow, 1962). Estos problemas restringen las posibilidades de acceder a fuentes de financiación externa (Annique y Montoro, 2010). Sin embargo, la literatura empírica no ha encontrado resultados concluyentes sobre la relación entre financiación pública e innovación. Según la revisión realizada por David et al. (2000), un tercio de los estudios sobre el tema encuentran que los subsidios a la I+D desplazan el gasto privado, aunque resalta que en las pequeñas y medianas empresas este factor es altamente relevante para explicar una mayor actividad innovadora.

Un último factor considerado es la antigüedad de la empresa, que ha sido incluido en varios estudios como una forma de aproximarse al efecto que sobre las capacidades tecnológicas pueden tener la acumulación de experiencia, el aprendizaje y también el posible estancamiento organizativo (Jefferson et al., 2006). Este aspecto puede ser de relevancia en el caso de las subsidiarias pues se ha encontrado que con el tiempo estas adquieren cada vez mayores capacidades tecnológicas (Berger y Diez, 2008).

\section{MODELO ECONOMÉTRICO Y FUENTES DE INFORMACIÓN}

El estudio hace uso del modelo planteado por Crépon, Duguet y Mairesse (1998, en adelante CDM), que permite interrelacionar el esfuerzo en actividades de innovación, la producción de innovaciones y la productividad de las empresas. Este modelo plantea un conjunto de ecuaciones que describen cada etapa del proceso de innovación y su posterior efecto sobre la productividad, permitiendo así corregir los sesgos de selección y la 
endogeneidad que median la relación entre estas dimensiones ${ }^{6}$. En una primera etapa, se examina la decisión de las empresas de involucrarse en actividades de innovación y la intensidad de la inversión en las mismas (es decir, la cantidad de recursos que se destinan para tal fin); en una segunda etapa se evalúa el impacto de la inversión en innovación sobre la producción de innovaciones (la llamada función de producción de conocimiento) y en una tercera etapa, se estima el efecto de la innovación sobre la productividad de las empresas. El modelo estructural y recursivo se compone de 4 ecuaciones interrelacionadas:

$$
\begin{aligned}
& y_{0 i}=\left\{\begin{array}{l}
1 \text { if } y_{0 i}^{*}=X_{0 i} \beta_{0}+\varepsilon_{0 i}>0 \\
0 \text { if } y_{0 i}^{*}=X_{0 i} \beta_{0}+\varepsilon_{0 i} \leq 0
\end{array}\right. \\
& y_{1 i}=\left\{\begin{array}{c}
y_{1 i}^{*}=X_{1 i} \beta_{1}+\varepsilon_{1 i} \text { if } y_{0 i}=1 \\
0 \text { if } y_{0 i}=0
\end{array}\right. \\
& y_{2 i}=\alpha_{1} \hat{y}_{1 i}+\beta_{2} X_{2 i}+\varepsilon_{2 i} \\
& y_{3 i}=\alpha_{3} \hat{y}_{2 i}+\beta_{i} X_{3 i}+\varepsilon_{3 i}
\end{aligned}
$$$$
\text { (Ecuación 1) }
$$$$
\text { (Ecuación 2) }
$$$$
\text { (Ecuación 3) }
$$$$
\text { (Ecuación 4) }
$$

Donde $y_{0 i}^{*}$ es una variable latente inobservada que representa la decisión de invertir en actividades de innovación, mientras que $y_{0 i}$ es una variable binaria asociada que toma valor 1 si las firmas invierten en Actividades de Innovación (AI) y 0 en otro caso. Las variables $y_{1 i}$, $y_{2 i}$ y $y_{3 i}$ hacen referencia a los insumos, los productos de la innovación y la productividad, respectivamente. Por su parte, $X_{0 i}, X_{1 i}, X_{2 i}$ y $X_{3 i}$ son los vectores de variables explicativas de las cuatro ecuaciones. Los $\beta_{j}$ y $\alpha_{j}$ son los vectores de parámetros de interés y los $\varepsilon_{j i}$ son los términos de error de las ecuaciones.

Las dos primeras ecuaciones se estiman conjuntamente mediante un modelo Tobit generalizado por máxima verosimilitud (Tobit tipo II usando el procedimiento de selección de Heckman (1979). En la estimación de la ecuación 3, se utilizan variables instrumentales y un modelo probit por máxima verosimilitud cuando se estiman los determinantes de la obtención de innovaciones de producto y proceso, y una regresión Tobit cuando la variable dependiente es el índice de innovación de producto. Por último, la ecuación 4 , se estima mediante un modelo que combina variables instrumentales y Mínimos Cuadrados en dos Etapas (2SLS) ${ }^{7}$. En el cuadro 1 se muestran las variables dependientes incluidas en el modelo, en el cuadro 2 los regresores incorporados en las diferentes etapas del modelo.

Para las estimaciones de las ecuaciones 1 a 3 se contemplan dos tipos de modelo dependiendo de si se tiene como variable dependiente la intensidad de la inversión en I+D (M1) o la intensidad del gasto en actividades de innovación (AI) (M2). La descripción detallada de las variables incluidas se encuentra en el anexo $1^{8}$.

\footnotetext{
${ }^{6}$ Los sesgos de selección se asocian a que sólo un número reducido de firmas realizan o registran gastos en I+D o en actividades de innovación. La simultaneidad se refiere a que los gastos en innovación son endógenos a la producción de innovaciones y esta última es endógena a la productividad.

7 El cálculo de las PTF se basa en la metodología propuesta por Levinsohn y Petrin (2003) la cual permite corregir los problemas de endogeneidad derivados la correlación que existe entre los shocks de productividad inobservados y los niveles de insumos utilizados en la producción.

${ }^{8}$ En el caso de las variables de sector y posición de las subsidiarias en su grupo empresarial, no se incluyen en las estimaciones las variables "Dominados por proveedores" y "Posición baja en el grupo", es decir, son consideradas categorías de referencia para evitar problemas de multicolinealidad perfecta.
} 


\begin{tabular}{|l|l|l|l|}
\hline \multicolumn{3}{|c|}{ CUADRO 1: VARIABLES DEPENDIENTES } \\
$\qquad$\begin{tabular}{|l|l|l|}
\hline Etapa & Variable & Indicadores \\
\hline I & $y_{0 i}$ & $\begin{array}{l}\text { Probabilidad de invertir en I+D total (M1) } \\
\text { Probabilidad de invertir en actividades de innovación - AI } \\
(\mathrm{M} 2)\end{array}$ \\
\hline II & $y_{1 i}$ & $\begin{array}{l}\text { Intensidad de la inversión en I+D (M1) } \\
\text { Intensidad de la inversión en actividades de innovación -AI } \\
\text { (M2) }\end{array}$ \\
\hline III & $y_{2 i}$ & $\begin{array}{l}\text { Innovación de proceso } \\
\text { Innovación de producto } \\
\text { Índice de la innovación de producto }\end{array}$ \\
\hline
\end{tabular} \\
\hline
\end{tabular}

\begin{tabular}{|c|c|c|c|c|c|}
\hline \multicolumn{6}{|c|}{ CUADRO 2: VARIABLES INDEPENDIENTES } \\
\hline \multirow{2}{*}{ Dimensión } & \multirow{2}{*}{ Indicadores } & \multicolumn{4}{|c|}{ Ecuación del modelo } \\
\hline & & I & II & III & IV \\
\hline \multirow{4}{*}{ Características de la empresa } & Cuota de mercado & $\mathrm{x}$ & $\mathrm{x}$ & & \\
\hline & Antigüedad & $\mathrm{x}$ & $\mathrm{x}$ & $\mathrm{x}$ & $\mathrm{x}$ \\
\hline & Personal $(\log )$ & $\mathrm{x}$ & & $\mathrm{x}$ & $\mathrm{x}$ \\
\hline & Capital físico & & & & $\mathrm{x}$ \\
\hline Apoyo público & Financiación pública AI & $\mathrm{x}$ & $\mathrm{x}$ & $\mathrm{x}$ & \\
\hline \multirow{2}{*}{ Apropiación del conocimiento } & Protección formal & $\mathrm{x}$ & $\mathrm{x}$ & $\mathrm{x}$ & \\
\hline & Protección estratégica & $\mathrm{x}$ & $\mathrm{x}$ & $\mathrm{x}$ & \\
\hline \multirow{6}{*}{ Cooperación } & Cooperación en I+D o AI & & $\mathrm{x}$ & & \\
\hline & Grupo & & & $\mathrm{x}$ & \\
\hline & Clientes & & & $\mathrm{x}$ & \\
\hline & Proveedores & & & $\mathrm{x}$ & \\
\hline & Competidores & & & $\mathrm{x}$ & \\
\hline & Universidades/Centros de I+D & & & $\mathrm{x}$ & \\
\hline \multirow{5}{*}{ Fuentes de ideas para innovar } & Grupo & & $\mathrm{x}$ & & \\
\hline & Clientes & & $\mathrm{x}$ & & \\
\hline & Proveedores & & $\mathrm{x}$ & & \\
\hline & Competidores & & $\mathrm{x}$ & & \\
\hline & Universidades/Centros de I+D & & $\mathrm{x}$ & & \\
\hline \multirow{4}{*}{ Obstáculos a la innovación } & Limitaciones de recursos & $\mathrm{x}$ & & & \\
\hline & Ausencia de personal cualificado & $\mathrm{x}$ & & & \\
\hline & Ausencia de información sobre & $\mathrm{x}$ & & & \\
\hline & Ausencia de información de mercado & $\mathrm{x}$ & & & \\
\hline \multirow{4}{*}{ Sector } & Basado en ciencia & $\mathrm{x}$ & $\mathrm{x}$ & $\mathrm{x}$ & $\mathrm{x}$ \\
\hline & Intensivo en escala & $\mathrm{x}$ & $\mathrm{x}$ & $\mathrm{x}$ & $\mathrm{x}$ \\
\hline & Proveedores especializados & $\mathrm{x}$ & $\mathrm{x}$ & $\mathrm{x}$ & $\mathrm{x}$ \\
\hline & Dominados por proveedores (referencia) & $\mathrm{x}$ & $\mathrm{x}$ & $\mathrm{x}$ & $\mathrm{x}$ \\
\hline \multirow{3}{*}{$\begin{array}{l}\text { Posición de las subsidiarias en la } \\
\text { corporación multinacional ( sólo para } \\
\text { subsidiarias) }\end{array}$} & Posición alta en su grupo & $\mathrm{x}$ & $\mathrm{x}$ & $\mathrm{x}$ & $\mathrm{x}$ \\
\hline & Posición media en su grupo & $\mathrm{x}$ & $\mathrm{x}$ & $\mathrm{x}$ & $\mathrm{x}$ \\
\hline & Posición baja en su grupo (referencia) & $\mathrm{x}$ & $\mathrm{x}$ & $\mathrm{x}$ & $\mathrm{x}$ \\
\hline $\begin{array}{l}\text { Aprendizaje mediante la exportación } \\
\text { ( sólo para exportadoras) }\end{array}$ & Continuidad exportación & $\mathrm{x}$ & $\mathrm{x}$ & $\mathrm{x}$ & $\mathrm{x}$ \\
\hline
\end{tabular}


Las fuentes de información utilizadas para las estimaciones son la Encuesta de Desarrollo e Innovación Tecnológica en su quinta versión (EDIT V) y la Encuesta Anual Manufacturera-EAM-, ambas emprendidas por el Departamento Administrativo Nacional de Estadísticas (Dane). La EDIT V recoge información censal para los años 2009 y 2010 de 8.243 empresas, aplicándose al conjunto de las firmas incluidas en el directorio de la EAM. Al integrar las dos bases de datos señaladas se obtuvo información tanto del proceso innovador como de indicadores que dan cuenta del desempeño productivo de las firmas manufactureras colombianas.

Una vez cruzados los datos de la EAM con los de la EDIT V y depurarla la información, obtuvimos finalmente 7.824 observaciones para realizar las estimaciones. De ellas 384 son subsidiarias de empresas extranjeras, 1.588 son empresas domésticas exportadoras y 5.712 son empresas domésticas no internacionalizadas.

\section{RESULTADOS}

\subsection{Realización de actividades de innovación}

En el cuadro 3 se presentan los resultados de la estimación de los determinantes de la decisión y de la intensidad de la inversión en I+D y en actividades de innovación por tipo de empresa, ya sea subsidiaria, doméstica exportadora o doméstica no exportadora. Se observa que el estimador Rho, mediante el cual se prueba la existencia de un sesgo de selección entre la probabilidad y la intensidad del gasto, es estadísticamente significativo para las subsidiarias y las empresas domésticas que no exportan, por lo que se justifica usar el método de Heckman (1979). En el caso de las empresas domésticas exportadoras es suficiente estimar por separado la intensidad de la inversión mediante Mínimos Cuadrados Ordinarios (MCO) y la probabilidad de invertir mediante un modelo Probit ${ }^{9}$.

En general, los resultados de las estimaciones muestran rasgos diferenciadores y también comunes en los determinantes de la probabilidad y la intensidad del gasto en I+D y en actividades de innovación en los tipos de empresas estudiados. El primer hecho que resalta es que la antigüedad de las empresas y la cuota de mercado no tienen relevancia para explicar la intensidad y la probabilidad del gasto en I+D y en AI, tanto para las subsidiarias como para las domésticas exportadoras, aunque sí lo tiene para las empresas domésticas no internacionalizadas. Por su parte, la probabilidad de que una empresa decida realizar esfuerzos en innovación se incrementa significativamente con el tamaño empresarial en todos los tipos de empresas (con excepción de la subsidiarias en el caso de la I+D). Esto guarda concordancia con las hipótesis Schumpeterianas que establecen que a mayor tamaño las empresas desarrollan ventajas relacionadas con el acceso a la financiación, economías de escala o una mejor organización del trabajo.

Para las empresas domésticas exportadoras y las subsidiarias, la vinculación de personal cualificado (técnicos y profesionales) tiene un impacto importante sobre la intensidad de la inversión en actividades de I+D y AI, pero tan sólo en las domésticas exportadoras aumenta la probabilidad de realizar ambos tipos de gasto. Por el contrario, el efecto del personal altamente cualificado (maestría y doctorado) tiene un efecto estadísticamente significativo sobre la probabilidad de invertir pero no sobre la intensidad del gasto en I+D y en AI, para todos los tipos de empresa.

\footnotetext{
${ }^{9}$ Para mayor detalle ver Greene (1999, p. 780) y Cameron \& Trivedi (2009, p. 541).
} 


\begin{tabular}{|c|c|c|c|c|c|c|c|c|c|c|c|c|}
\hline \multicolumn{13}{|c|}{$\begin{array}{c}\text { CUADRO 3: ESTIMACIÓN DE LA DECISIÓN Y DE LA INTENSIDAD DE LA INVERSIÓN EN I+D } \\
\text { Y EN ACTIVIDADES DE INNOVACIÓN }\end{array}$} \\
\hline & \multicolumn{4}{|c|}{ Subsidiarias } & \multicolumn{4}{|c|}{ Exportadoras } & \multicolumn{4}{|c|}{ Domesticas no exportadoras } \\
\hline & \multicolumn{2}{|c|}{ I+D } & \multicolumn{2}{|c|}{$\mathbf{A}$} & \multicolumn{2}{|c|}{ I+D } & \multicolumn{2}{|c|}{$\mathbf{A}$} & \multicolumn{2}{|c|}{ I+D } & \multicolumn{2}{|c|}{$\mathbf{A}$} \\
\hline & Prob & Int & Prob & Int & Prob & Int & Prob & Int & Prob & Int & Prob & Int \\
\hline $\begin{array}{l}\text { Características de la empresa } \\
\text { Antigüedad }\end{array}$ & $\begin{array}{l}-0,007 \\
(0,027)\end{array}$ & $\begin{array}{c}0,046 \\
(0,286)\end{array}$ & $\begin{array}{r}-0,039 \\
(0,033)\end{array}$ & $\begin{array}{c}0,188 \\
(0,150)\end{array}$ & $\begin{array}{c}0,004 \\
(0,014)\end{array}$ & $\begin{array}{r}-0,192 \\
(0,143)\end{array}$ & $\begin{array}{l}-0,008 \\
(0,020)\end{array}$ & $\begin{array}{r}-0,099 \\
(0,075)\end{array}$ & $\begin{array}{l}-0,006 \\
(0,004)\end{array}$ & $\begin{array}{l}-0.229^{* *} \\
(0,098)\end{array}$ & $\begin{array}{c}-0.058^{* * *} \\
(0,010)\end{array}$ & $\begin{array}{c}-0.117^{\star *} \\
(0,054)\end{array}$ \\
\hline Cuota de mercado & $\begin{array}{c}1,893 \\
-1,356\end{array}$ & $\begin{array}{c}4,879 \\
-20,658\end{array}$ & $\begin{array}{c}0,46 \\
-1,959\end{array}$ & $\begin{array}{l}7,859 \\
-7,084\end{array}$ & $\begin{array}{l}0,834 \\
-0,9\end{array}$ & $\begin{array}{c}0,108 \\
-6,114\end{array}$ & $\begin{array}{c}0,252 \\
-1,303\end{array}$ & $\begin{array}{c}6.706^{* *} \\
-3,27\end{array}$ & $\begin{array}{c}0,498 \\
-0,5\end{array}$ & $\begin{array}{l}1,536 \\
-3,981\end{array}$ & $\begin{array}{l}1,625 \\
-1,495\end{array}$ & $\begin{array}{l}7.073^{*} \\
-4,229\end{array}$ \\
\hline Personal (log) & $\begin{array}{c}0,021 \\
(0,022)\end{array}$ & & $\begin{array}{c}0.072^{* * *} \\
(0,024)\end{array}$ & & $\begin{array}{c}0.036^{* * *} \\
(0,009)\end{array}$ & & $\begin{array}{l}0.111^{* * *} \\
(0,013)\end{array}$ & & $\begin{array}{c}0.021^{* * *} \\
(0,003)\end{array}$ & & $\begin{array}{l}0.124^{* * *} \\
(0,008)\end{array}$ & \\
\hline Recurso humano & & & & & & & & & & & & \\
\hline Personal cualificado & $\begin{array}{c}0,003 \\
(0,011)\end{array}$ & $\begin{array}{c}1.222^{* * *} \\
(0,291)\end{array}$ & $\begin{array}{c}0,041 \\
(0,032)\end{array}$ & $\begin{array}{c}0.674^{* * *} \\
(0,173)\end{array}$ & $\begin{array}{c}0.036^{* \star *} \\
(0,012)\end{array}$ & $\begin{array}{c}0.836^{* * *} \\
(0,154)\end{array}$ & $\begin{array}{c}0,006 \\
(0,006)\end{array}$ & $\begin{array}{c}0.077^{* * *} \\
(0,024)\end{array}$ & $\begin{array}{c}0.002^{\star \star \star} \\
(0,001)\end{array}$ & $\begin{array}{c}0,011 \\
(0,022)\end{array}$ & $\begin{array}{c}0.008^{\star * *} \\
(0,001)\end{array}$ & $\begin{array}{l}0.016^{\star} \\
(0,008)\end{array}$ \\
\hline $\begin{array}{l}\text { Personalla altamente cualificado } \\
\text { Apoyo público }\end{array}$ & $\begin{array}{l}0.003^{* *} \\
(0,001)\end{array}$ & $\begin{array}{l}-0,003 \\
(0,018)\end{array}$ & $\begin{array}{r}-0,002 \\
(0,002)\end{array}$ & $\begin{array}{l}0.014^{*} \\
(0,008)\end{array}$ & $\begin{array}{l}0.002^{*} \\
(0,001)\end{array}$ & $\begin{array}{c}0,011 \\
(0,009)\end{array}$ & $\begin{array}{l}0.003^{\star *} \\
(0,001)\end{array}$ & $\begin{array}{l}0.016^{* * *} \\
(0,004)\end{array}$ & $\begin{array}{l}0.001^{* * *} \\
0,000\end{array}$ & $\begin{array}{c}0,005 \\
(0,007)\end{array}$ & $\begin{array}{c}0.003^{* * *} \\
(0,001)\end{array}$ & $\begin{array}{c}0.013^{* * *} \\
(0,004)\end{array}$ \\
\hline Financiacion publica & $\begin{array}{c}0.593^{* \star *} \\
(0,200)\end{array}$ & $\begin{array}{l}1,214 \\
(0,997)\end{array}$ & $\begin{array}{c}0.224^{* \star *} \\
(0,036)\end{array}$ & $\begin{array}{c}0,569 \\
(0,660)\end{array}$ & $\begin{array}{c}0.400^{\star \star \star *} \\
(0,094)\end{array}$ & $\begin{array}{l}0.859^{*} \\
(0,456)\end{array}$ & $\begin{array}{c}0.450^{\star \star \star} \\
(0,016)\end{array}$ & $\begin{array}{c}0,394 \\
(0,242)\end{array}$ & $\begin{array}{c}0.193^{* * *} \\
(0,067)\end{array}$ & $\begin{array}{c}1.687^{* * *} \\
(0,432)\end{array}$ & $\begin{array}{l}0.684^{\star * *} \\
(0,007)\end{array}$ & $\begin{array}{c}1.217^{\star \star *} \\
(0,214)\end{array}$ \\
\hline $\begin{array}{l}\text { Apropiación del conocimien } \\
\text { Proteccion formal }\end{array}$ & 0,147 & 0,204 & 0,074 & $-0.761^{*}$ & $0.084^{*}$ & 0,216 & $0.092^{*}$ & $-0,067$ & & 0,067 & $0.133^{* * *}$ & $-0.151^{*}$ \\
\hline Proteccion estrategica & $\begin{array}{l}(0,106) \\
0,022\end{array}$ & $\begin{array}{l}(0,669) \\
-0,276\end{array}$ & $\begin{array}{l}(0,079) \\
0.086^{*}\end{array}$ & $\begin{array}{l}(0,429) \\
0.506^{\star \star}\end{array}$ & $\begin{array}{l}(0,043) \\
0.133^{* * \star}\end{array}$ & $\begin{array}{l}(0,323) \\
0,282\end{array}$ & $\begin{array}{l}(0,052) \\
0.196^{* *}\end{array}$ & $\begin{array}{l}(0,179) \\
0.418^{* \star \star}\end{array}$ & $\begin{array}{l}(0,008) \\
0.072^{\star \star \star}\end{array}$ & $\begin{array}{l}(0,173) \\
0.341^{*}\end{array}$ & $\begin{array}{l}(0,018) \\
0.177^{\star \star \star}\end{array}$ & $\begin{array}{l}(0,082) \\
0.365^{\star \star \star}\end{array}$ \\
\hline Cooperación & $(0,046)$ & $(0,565)$ & $(0,047)$ & $(0,246)$ & $(0,031)$ & $(0,272)$ & $(0,033)$ & $(0,137)$ & $(0,011)$ & $(0,181)$ & $(0,021)$ & $(0,083)$ \\
\hline & & $\begin{array}{c}0,382 \\
(0,439)\end{array}$ & & & & $\begin{array}{c}-0,03 \\
(0,201)\end{array}$ & & & & $\begin{array}{c}0,118 \\
(0,174)\end{array}$ & & \\
\hline Cooperacion en Al & & & & $\begin{array}{c}0,295 \\
(0,240)\end{array}$ & & & & $\begin{array}{c}0,141 \\
(0,124)\end{array}$ & & & & 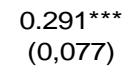 \\
\hline Fuentes de ideas para innovar & & & & & & & & & & & & \\
\hline Grupo & & $\begin{array}{c}0,458 \\
(0,360)\end{array}$ & & $\begin{array}{c}0,147 \\
(0,236)\end{array}$ & & $\begin{array}{c}0.907^{\star * *} \\
(0,243)\end{array}$ & & $\begin{array}{c}0.466^{\star \star \star} \\
(0,139)\end{array}$ & & $\begin{array}{c}0.535^{\star \star *} \\
(0,203)\end{array}$ & & $\begin{array}{c}0,149 \\
(0,145)\end{array}$ \\
\hline Clientes & & $\begin{array}{c}0,757 \\
(0,579)\end{array}$ & & $\begin{array}{c}0,188 \\
(0,226)\end{array}$ & & $\begin{array}{c}0,148 \\
(0,226)\end{array}$ & & $\begin{array}{c}0,003 \\
(0,115)\end{array}$ & & $\begin{array}{c}0,149 \\
(0,179)\end{array}$ & & $\begin{array}{c}0,065 \\
(0,081)\end{array}$ \\
\hline Proveedores & & $\begin{array}{c}0,428 \\
(0,442)\end{array}$ & & $\begin{array}{c}0,205 \\
(0,221)\end{array}$ & & $\begin{array}{r}-0,162 \\
(0,214)\end{array}$ & & $\begin{array}{c}0,100 \\
(0,119)\end{array}$ & & $\begin{array}{l}-0,038 \\
(0,158)\end{array}$ & & $\begin{array}{l}0.175^{\star \star} \\
(0,080)\end{array}$ \\
\hline Competidores & & $\begin{array}{c}0,082 \\
(0,393)\end{array}$ & & $\begin{array}{l}-0,014 \\
(0,236)\end{array}$ & & $\begin{array}{c}0,285 \\
(0,219)\end{array}$ & & $\begin{array}{c}0,015 \\
(0,120)\end{array}$ & & $\begin{array}{l}0,096 \\
(0,158)\end{array}$ & & $\begin{array}{l}0.174^{\star *} \\
(0,082)\end{array}$ \\
\hline Universidades/Centros I+D & & $\begin{array}{r}-0,300 \\
(0,561)\end{array}$ & & $\begin{array}{r}-0,142 \\
(0,294)\end{array}$ & & $\begin{array}{l}-0,099 \\
(0,204)\end{array}$ & & $\begin{array}{l}0.265^{\star *} \\
(0.125)\end{array}$ & & $\begin{array}{c}0,04 \\
(0,185)\end{array}$ & & $\begin{array}{c}0,004 \\
(0,104)\end{array}$ \\
\hline
\end{tabular}

Nota: * Significativo al 10 por $100 * *$ Significativo al 5 por $100 * * *$ Significativo al 1 por 100 . Las desviaciones típicas robustas que se encuentra en paréntesis. Se registran los efectos marginales en el caso de la probabilidad de invertir. 


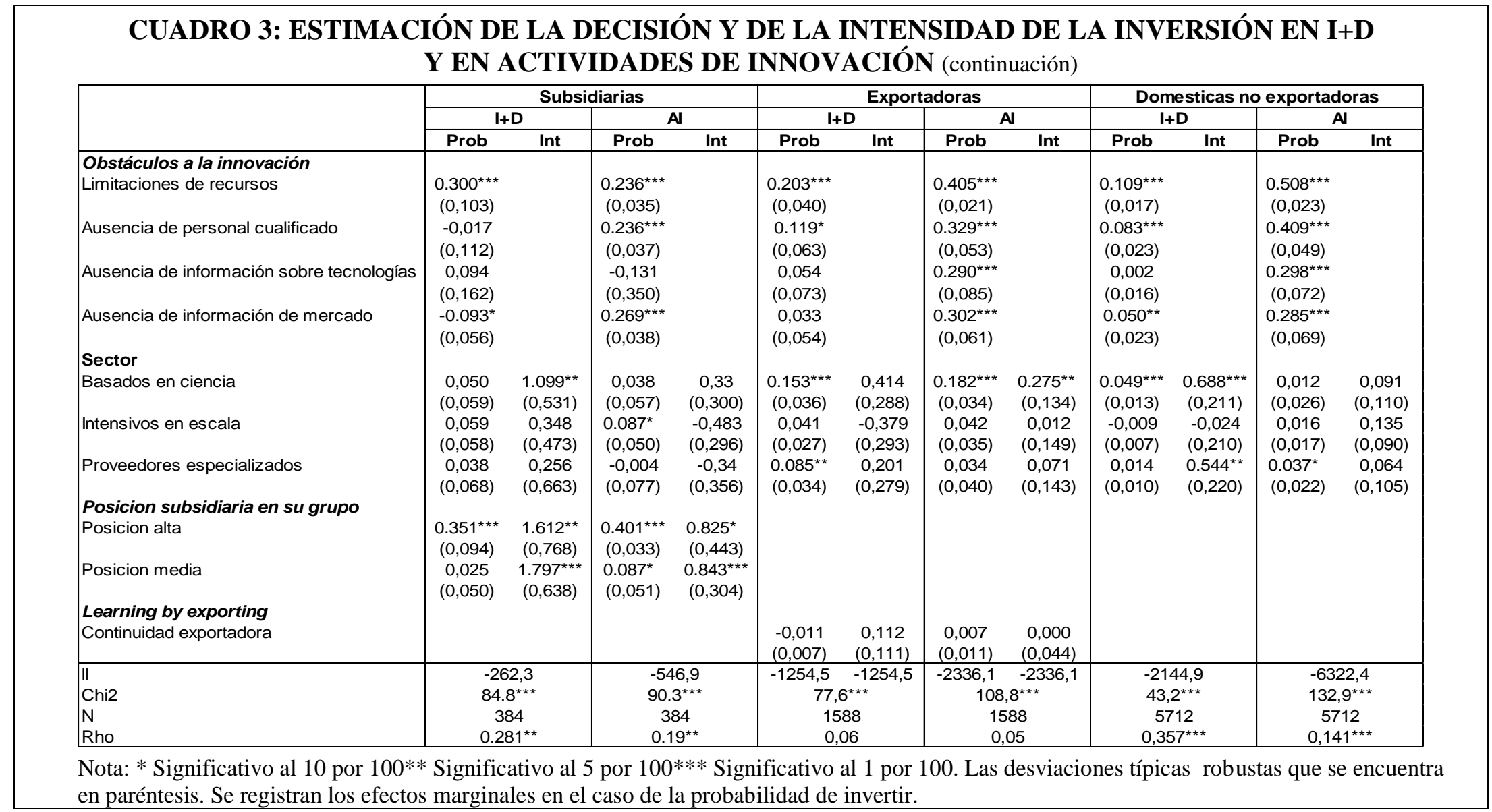


Los subsidios públicos dirigidos a financiar la innovación se advierten ampliamente relacionados con la probabilidad de invertir en I+D y en actividades de innovación para todas las empresas. Sin embargo, no se relacionan con su intensidad salvo para las empresas domésticas no exportadoras. En particular, en las subsidiarias que recibieron apoyo financiero por parte del gobierno la probabilidad de invertir en I+D se incrementa en un 60 por $100 \mathrm{y}$ un 22 por 100 cuando nos referimos a las AI, mientras que para las empresas domésticas exportadoras esta probabilidad alcanza el 40 por 100 y el 45 por 100, respectivamente.

En las subsidiarias extranjeras, los mecanismos estratégicos de protección de la innovación aumentan la probabilidad y la intensidad de realizar actividades de innovación pero no las de I+D. En contraste, en las empresas domésticas exportadoras tanto los mecanismos de protección formales como estratégicos impactan positivamente en la probabilidad de realizar I+D y AI, y también tienen un efecto sobre el esfuerzo en AI.

La cooperación en I+D no muestra efecto alguno sobre el esfuerzo en $\mathrm{I}+\mathrm{D}$, cualquiera que sea el tipo de empresa evaluada. Por su parte, la cooperación en AI se encuentra relacionada positivamente con la intensidad de la inversión en AI, únicamente en el caso de las empresas domésticas no internacionalizadas. Cuando se analizan las fuentes de ideas para la innovación, se observa que en el caso de las domésticas exportadoras los flujos de conocimiento provenientes de su grupo empresarial y con organizaciones de I+D tienen impacto sobre el esfuerzo en AI; en las empresas domésticas que no exportan es importante la información proveniente de proveedores y competidores y, por último, en las subsidiarias, ningún tipo de flujo de conocimiento tiene efecto sobre los insumos de la innovación.

El efecto de las variables asociadas a los obstáculos del proceso innovador muestran un patrón similar entre la domésticas exportadoras y las subsidiarias, siendo las limitaciones de recursos el factor con mayor incidencia, sin importar si se trata de realizar I+D o AI. En el caso de las domésticas exportadoras y de la inversión en AI se observa un efecto importante de otros obstáculos como la ausencia de personal cualificado y la falta de información de mercado.

A nivel sectorial, las subsidiarias vinculadas a sectores intensivos en escala tienen una mayor probabilidad de invertir en $\mathrm{AI}$, mientras que la probabilidad de invertir en $\mathrm{I}+\mathrm{D}$ es mayor en industrias dominadas por los proveedores. En el caso de las domésticas exportadoras el hecho de pertenecer a sectores basados en la ciencia, donde existen mayores oportunidades tecnológicas, es mucho más relevante para explicar la decisión de invertir tanto en I+D como en actividades de innovación. También es significativo para el grupo de empresas exportadoras la pertenencia de las firmas al sector de proveedores especializados.

Al evaluar el efecto de los regresores específicos diseñados para la posición de las subsidiarias dentro de su grupo, se observa que la probabilidad de realizar actividades de I+D y AI crece en la medida que estas unidades detentan una mejor posición en su corporación multinacional. Es decir, cuando las subsidiarias mantienen una orientación hacia los mercados internacionales y tienen una unidad formal de $\mathrm{I}+\mathrm{D}$, es mayor la probabilidad y el esfuerzo innovador, siendo mayor el efecto en el caso de la inversión en AI en comparación a la I+D. En cuanto al efecto de los procesos de aprendizaje mediante la exportación, no se observa un efecto robusto sobre los insumos de la innovación.

\subsection{Innovación}

En el cuadro 4, se presentan los resultados de la estimación de la función de producción de conocimiento. En ella se encuentran reflejados los efectos marginales y la desviación estándar robusta del modelo Probit para las innovaciones de producto y de proceso, mientras 
que en el caso del índice de producto construido se muestran los coeficientes obtenidos al utilizar un modelo Tobit.

Para todos los tipos de empresas analizados, la intensidad del gasto total en actividades de innovación -AI- predicha en la primera etapa del modelo, tiene un efecto positivo y significativo sobre la probabilidad de obtener innovaciones de producto, de proceso y sobre el índice de producto. En contraste, el esfuerzo en I+D tiene efectos robustos sobre todas las medidas de innovación en las empresas nacionales exportadoras y no exportadoras, pero en las subsidiarias tan sólo tiene impacto en la innovación de proceso. Esto sugiere que el esfuerzo en I+D de las subsidiarias no se traduce en una mayor producción de innovaciones de producto, y que en el caso de haberse obtenido han sido el resultado de desarrollar otras actividades de innovación diferentes a la $\mathrm{I}+\mathrm{D}$ con menor valor en términos tecnológicos, como puede ser la compra de maquinaria y equipo.

En concreto, para las subsidiarias la probabilidad de obtener innovaciones de proceso se incrementa un 7,7 por 100 con la realización de actividades de I+D y un 3,7 por 100 con la ejecución de actividades de innovación; así por 100mismo la inversión en AI aumenta en un 4,1 por 100 la probabilidad de obtener innovaciones de producto. En las nacionales exportadoras, el rendimiento en términos de la producción de innovaciones tecnológicas es mayor cuando se invierte en I+D que en AI: las nacionales exportadoras que ejecutan esfuerzos en I+D tienen 7,7\% más probabilidad de obtener innovaciones de producto y un 11 por 100 en proceso, mientras que el rendimiento del esfuerzo en AI es del 5,2 por 100 para nuevos productos y del 6,7 por 100 para la innovación de proceso.

En cuanto a la antigüedad, se encontró que tan sólo las empresas domésticas no internacionalizadas de más reciente creación poseen una mayor probabilidad de obtener innovaciones de producto y proceso, incluyendo las de alta novedad cuando se invierte en I+D (M1). En las empresas internacionalizadas no se encuentra relación entre la producción de innovaciones y la antigüedad de la empresa. Otras variables con efectos no esperados son la cuota de mercado y las variables asociadas a la cualificación del personal, para las cuales no se encontraron efectos estadísticamente significativos. El efecto marginal asociado al tamaño de la empresa tiene el signo esperado y es estadísticamente significativo, en especial para la innovación de producto y el modelo 1 (M1) en todos los tipos de empresa.

Los subsidios públicos para financiar actividades de innovación ya no se advierten tan relevantes para explicar la producción de innovaciones, como sí se observaba en la etapa anterior del modelo. Para las subsidiarias, el efecto de esta variable es positivo sobre la innovación de producto pero negativo para la innovación de proceso, mientras que para las domésticas exportadoras su impacto es negativo en la innovación de proceso y no tiene efecto sobre la innovación de producto.

En las empresas extranjeras, los mecanismos de protección formales son los que tienen mayor relación con la producción de innovaciones de producto y de proceso. En contraste, en las firmas nacionales exportadoras tanto los mecanismos formales como estratégicos de protección aumentan la probabilidad de innovar, en especial hacia la innovación de producto.

En lo que las relaciones con otras organizaciones se refiere, la cooperación con proveedores es la única variable con un efecto significativo y positivo en la obtención de todo tipo de innovaciones y para todos los grupos analizados. La cooperación con clientes es de relevancia para la innovación de producto y proceso en las empresas domésticas tanto para las que exportan como para las que no, pero no tanto para las subsidiarias extranjeras en las que es más importante la cooperación con proveedores. 


\begin{tabular}{|c|c|c|c|c|c|c|c|c|c|c|c|c|c|c|c|c|c|c|}
\hline \multicolumn{19}{|c|}{ CUADRO 4: ESTIMACIÓN DE LA FUNCIÓN DE PRODUCCIÓN DE CONOCIMIENTO POR TIPO DE INNOVACIÓN } \\
\hline & \multicolumn{6}{|c|}{\begin{tabular}{|c|} 
Subsidiarias \\
\end{tabular}} & \multicolumn{6}{|c|}{\begin{tabular}{|l|} 
Exportadoras \\
\end{tabular}} & \multicolumn{6}{|c|}{\begin{tabular}{|c|} 
Domésticas no exportadoras \\
\end{tabular}} \\
\hline & \multicolumn{2}{|c|}{$\begin{array}{l}\text { Innovación de } \\
\text { proceso }\end{array}$} & \multicolumn{2}{|c|}{$\begin{array}{l}\text { Innovación de } \\
\text { producto }\end{array}$} & \multicolumn{2}{|c|}{$\begin{array}{l}\text { Índice de } \\
\text { innovación } \\
\text { producto }\end{array}$} & \multicolumn{2}{|c|}{$\begin{array}{l}\text { Innovación de } \\
\text { proceso }\end{array}$} & \multicolumn{2}{|c|}{$\begin{array}{l}\text { Innovación de } \\
\text { producto }\end{array}$} & \multicolumn{2}{|c|}{$\begin{array}{c}\text { Índice de } \\
\text { innovación } \\
\text { producto }\end{array}$} & \multicolumn{2}{|c|}{$\begin{array}{c}\text { Innovación de } \\
\text { proceso }\end{array}$} & \multicolumn{2}{|c|}{$\begin{array}{l}\text { Innovación de } \\
\text { producto }\end{array}$} & \multicolumn{2}{|c|}{$\begin{array}{c}\text { Índice de } \\
\text { innovación } \\
\text { producto }\end{array}$} \\
\hline & M1 & M2 & M1 & M2 & M1 & M2 & M1 & M2 & M1 & M2 & M1 & M2 & M1 & M2 & M1 & M2 & M1 & M2 \\
\hline $\begin{array}{l}\text { Insumos de la innovación } \\
\text { I+D (predicha en etapa I) }\end{array}$ & $\begin{array}{l}0.077^{*} \\
(0,040)\end{array}$ & & $\begin{array}{l}-0,034 \\
(0,046)\end{array}$ & & $\begin{array}{l}-0,047 \\
(0,054)\end{array}$ & & $\begin{array}{c}0.113^{\star \star *} \\
(0,023)\end{array}$ & & $\begin{array}{c}0.077^{\star \star *} \\
(0,023)\end{array}$ & & $\begin{array}{l}0.062^{* *} \\
(0,029)\end{array}$ & & $\begin{array}{c}0.095^{* * *} \\
(0,015)\end{array}$ & & $\begin{array}{c}0.088^{* * *} \\
(0,011)\end{array}$ & & $\begin{array}{c}0.201^{* * *} \\
(0,026)\end{array}$ & \\
\hline Al (predicha en etapa l) & & $\begin{array}{l}0.037^{\star *} \\
(0,017)\end{array}$ & & $\begin{array}{l}0.041^{* *} \\
(0,017)\end{array}$ & & $\begin{array}{l}0.051^{* *} \\
(0,024)\end{array}$ & & $\begin{array}{c}0.067^{\star \star *} \\
(0,009)\end{array}$ & & $\begin{array}{c}0.052^{\star \star \star} \\
(0,009)\end{array}$ & & $\begin{array}{c}0.054^{\star \star *} \\
(0,011)\end{array}$ & & $\begin{array}{c}0.055^{\star \star \star} \\
(0,004)\end{array}$ & & $\begin{array}{c}0.038^{\star * \star} \\
(0,003)\end{array}$ & & $\begin{array}{c}0.118^{\star \star \star} \\
(0,008)\end{array}$ \\
\hline $\begin{array}{l}\text { Característic } \\
\text { Antigüedad }\end{array}$ & $\begin{array}{c}0,017 \\
(0,041)\end{array}$ & $\begin{array}{c}0,019 \\
(0,041)\end{array}$ & $\begin{array}{c}0,019 \\
(0,042)\end{array}$ & $\begin{array}{c}0,025 \\
(0,042)\end{array}$ & $\begin{array}{c}0,031 \\
(0,042)\end{array}$ & $\begin{array}{c}0,035 \\
(0,042)\end{array}$ & $\begin{array}{c}0,013 \\
(0,019)\end{array}$ & $\begin{array}{c}0,02 \\
(0,019)\end{array}$ & $\begin{array}{c}0,01 \\
(0,019)\end{array}$ & $\begin{array}{c}0,016 \\
(0,019)\end{array}$ & $\begin{array}{l}-0,009 \\
(0,021)\end{array}$ & $\begin{array}{l}-0,004 \\
(0,022)\end{array}$ & $\begin{array}{c}-0.042^{* \star *} \\
(0,008)\end{array}$ & $\begin{array}{c}-0.023^{\star * \star} \\
(0,008)\end{array}$ & $\begin{array}{l}-0.010^{*} \\
(0,006)\end{array}$ & $\begin{array}{c}0,002 \\
(0,006)\end{array}$ & $\begin{array}{l}-0.036^{*} \\
(0,020)\end{array}$ & $\begin{array}{c}0,004 \\
(0,020)\end{array}$ \\
\hline Cuota de mercado & $\begin{array}{l}-0,016 \\
(0,022)\end{array}$ & $\begin{array}{l}-0,014 \\
(0,022)\end{array}$ & $\begin{array}{l}-0,014 \\
(0,024)\end{array}$ & $\begin{array}{l}-0,021 \\
(0,024)\end{array}$ & $\begin{array}{l}-0,034 \\
(0,023)\end{array}$ & $\begin{array}{l}-0.046^{*} \\
(0,025)\end{array}$ & $\begin{array}{c}-5.085^{\star \star \star} \\
(1,832)\end{array}$ & $\begin{array}{l}-3.783^{* *} \\
(1,496)\end{array}$ & $\begin{array}{l}-2.766^{*} \\
(1,562)\end{array}$ & $\begin{array}{l}-2.218^{*} \\
(1,316)\end{array}$ & $\begin{array}{l}-1,581 \\
(2,330)\end{array}$ & $\begin{array}{l}-1,095 \\
(2,063)\end{array}$ & $\begin{array}{l}-0,571 \\
(1,123)\end{array}$ & $\begin{array}{l}-0,427 \\
(1,028)\end{array}$ & $\begin{array}{c}0,952 \\
(0,693)\end{array}$ & $\begin{array}{l}1.212^{*} \\
(0,659)\end{array}$ & $\begin{array}{l}2.328^{\star} \\
(1,203)\end{array}$ & $\begin{array}{c}2.791^{* * *} \\
(0,897)\end{array}$ \\
\hline $\begin{array}{l}\text { Personal (log) } \\
\text { Recurso humano }\end{array}$ & $\begin{array}{c}0,014 \\
(0,034)\end{array}$ & $\begin{array}{c}0,007 \\
(0,035)\end{array}$ & $\begin{array}{l}0.065^{*} \\
(0,037)\end{array}$ & $\begin{array}{c}0,045 \\
(0,037)\end{array}$ & $\begin{array}{l}0.088^{\star \star} \\
(0,042)\end{array}$ & $\begin{array}{c}0,067 \\
(0,044)\end{array}$ & $\begin{array}{l}0.023^{*} \\
(0,013)\end{array}$ & $\begin{array}{l}-0,004 \\
(0,014)\end{array}$ & $\begin{array}{l}0.046^{\star * *} \\
(0,012)\end{array}$ & $\begin{array}{l}0.025^{*} \\
(0,013)\end{array}$ & $\begin{array}{c}0.059^{\star \star *} \\
(0,016)\end{array}$ & $\begin{array}{l}0.038^{\star *} \\
(0,017)\end{array}$ & $\begin{array}{l}0.051^{* \star *} \\
(0,006)\end{array}$ & $\begin{array}{c}0.024^{\star \star \star} \\
(0,006)\end{array}$ & $\begin{array}{c}0.021^{* \star *} \\
(0,005)\end{array}$ & $\begin{array}{c}0,007 \\
(0,005)\end{array}$ & $\begin{array}{l}0.083^{\star \star \star} \\
(0,015)\end{array}$ & $\begin{array}{l}0.032^{* *} \\
(0,015)\end{array}$ \\
\hline$\%$ Personal cualificado & $\begin{array}{c}0,003 \\
(0,008)\end{array}$ & $\begin{array}{l}-0,001 \\
(0,007)\end{array}$ & $\begin{array}{c}0,005 \\
(0,015)\end{array}$ & $\begin{array}{l}-0,006 \\
(0,007)\end{array}$ & $\begin{array}{c}0,001 \\
(0,014)\end{array}$ & $\begin{array}{l}-0,012 \\
(0,009)\end{array}$ & $\begin{array}{l}-0,005 \\
(0,004)\end{array}$ & $\begin{array}{l}-0,005 \\
(0,004)\end{array}$ & $\begin{array}{l}0.013^{\star *} \\
(0,006)\end{array}$ & $\begin{array}{c}0,025 \\
(0,015)\end{array}$ & $\begin{array}{l}0.020^{*} \\
(0,011)\end{array}$ & $\begin{array}{c}0,033 \\
(0,022)\end{array}$ & $\begin{array}{c}0.004^{* \star *} \\
(0,001)\end{array}$ & $\begin{array}{l}0.002^{* *} \\
(0,001)\end{array}$ & $\begin{array}{c}0.004^{\star \star *} \\
(0,001)\end{array}$ & $\begin{array}{c}0.002^{\star \star *} \\
(0,001)\end{array}$ & $\begin{array}{c}0.013^{\star \star *} \\
(0,003)\end{array}$ & $\begin{array}{c}0.009^{* * *} \\
(0,003)\end{array}$ \\
\hline $\begin{array}{l}\text { \% Personal altamente cualificado } \\
\text { Apoyo público }\end{array}$ & $\begin{array}{c}0,002 \\
(0,002)\end{array}$ & $\begin{array}{c}0,003 \\
(0,002)\end{array}$ & $\begin{array}{c}0,000 \\
(0,002)\end{array}$ & $\begin{array}{l}-0,001 \\
(0,002)\end{array}$ & $\begin{array}{c}0,000 \\
(0,002)\end{array}$ & $\begin{array}{l}-0,001 \\
(0,002)\end{array}$ & $\begin{array}{l}-0,001 \\
(0,001)\end{array}$ & $\begin{array}{l}-0,001 \\
(0,001)\end{array}$ & $\begin{array}{c}0,000 \\
(0,001)\end{array}$ & $\begin{array}{c}0,000 \\
(0,001)\end{array}$ & $\begin{array}{c}0,000 \\
(0,001)\end{array}$ & $\begin{array}{c}0,000 \\
(0,001)\end{array}$ & $\begin{array}{l}-0.001^{*} \\
(0,001)\end{array}$ & $\begin{array}{l}-0,001 \\
(0,001)\end{array}$ & $\begin{array}{c}0,000 \\
(0,001)\end{array}$ & $\begin{array}{c}0,000 \\
(0,001)\end{array}$ & $\begin{array}{l}-0,001 \\
(0,002)\end{array}$ & $\begin{array}{c}0,000 \\
(0,002)\end{array}$ \\
\hline Financiación pública Al & $\begin{array}{c}-0.226^{\star *} \\
(0,095)\end{array}$ & $\begin{array}{l}-0,142 \\
(0,132)\end{array}$ & $\begin{array}{c}0.548^{\star \star *} \\
(0,185)\end{array}$ & $\begin{array}{c}0,272 \\
(0,267)\end{array}$ & $\begin{array}{c}0,291 \\
(0,243)\end{array}$ & $\begin{array}{l}-0,041 \\
(0,152)\end{array}$ & $\begin{array}{c}-0.177^{\star *} \\
(0,078)\end{array}$ & $\begin{array}{c}-0,09 \\
(0,078)\end{array}$ & $\begin{array}{c}0,043 \\
(0,130)\end{array}$ & $\begin{array}{c}0,08 \\
(0,105)\end{array}$ & $\begin{array}{c}0,09 \\
(0,148)\end{array}$ & $\begin{array}{c}0,116 \\
(0,111)\end{array}$ & $\begin{array}{l}-0,087 \\
(0,055)\end{array}$ & $\begin{array}{c}-0.102^{\star \star \star} \\
(0,034)\end{array}$ & $\begin{array}{c}-0,03 \\
(0,046)\end{array}$ & $\begin{array}{l}-0,017 \\
(0,038)\end{array}$ & $\begin{array}{l}-0,033 \\
(0,161)\end{array}$ & $\begin{array}{l}-0,041 \\
(0,120)\end{array}$ \\
\hline Protección formal & $\begin{array}{c}0,072 \\
(0,054)\end{array}$ & $\begin{array}{l}0.116^{*} \\
(0,059)\end{array}$ & $\begin{array}{c}0.169^{\star \star \star} \\
(0,059)\end{array}$ & $\begin{array}{c}0.240^{* * *} \\
(0,063)\end{array}$ & $\begin{array}{c}3.542^{\star \star *} \\
(1,230)\end{array}$ & $\begin{array}{c}5.374^{\star \star \star} \\
(1,350)\end{array}$ & $\begin{array}{l}0.060^{*} \\
(0,036)\end{array}$ & $\begin{array}{c}0,017 \\
(0,033)\end{array}$ & $\begin{array}{l}0.080^{* *} \\
(0,035)\end{array}$ & $\begin{array}{c}0,026 \\
(0,032)\end{array}$ & $\begin{array}{l}1.766^{* *} \\
(0,773)\end{array}$ & $\begin{array}{c}0,502 \\
(0,700)\end{array}$ & $\begin{array}{c}0.045^{* \star *} \\
(0,015)\end{array}$ & $\begin{array}{c}0.115^{\star \star \star} \\
(0,017)\end{array}$ & $\begin{array}{c}0.054^{\star \star \star} \\
(0,012)\end{array}$ & $\begin{array}{c}0.109^{\star \star \star} \\
(0,015)\end{array}$ & $\begin{array}{c}1.483^{\star * \star} \\
(0,308)\end{array}$ & $\begin{array}{c}3.126^{\star \star \star} \\
(0,342)\end{array}$ \\
\hline $\begin{array}{l}\text { Protección estratégica } \\
\text { Cooperación }\end{array}$ & $\begin{array}{l}0.112^{* *} \\
(0,055)\end{array}$ & $\begin{array}{l}-0,062 \\
(0,098)\end{array}$ & $\begin{array}{c}0,096 \\
(0,059)\end{array}$ & $\begin{array}{c}-0.221^{* *} \\
(0,098)\end{array}$ & $\begin{array}{l}2.435^{\star} \\
(1,258)\end{array}$ & $\begin{array}{c}-5.428^{* *} \\
(2,333)\end{array}$ & $\begin{array}{c}0,044 \\
(0,034)\end{array}$ & $\begin{array}{c}-0.125^{\star \star \star} \\
(0,046)\end{array}$ & $\begin{array}{c}0.142^{* * *} \\
(0,034)\end{array}$ & $\begin{array}{l}-0,028 \\
(0,045)\end{array}$ & $\begin{array}{c}3.422^{* * *} \\
(0,746)\end{array}$ & $\begin{array}{l}-0,821 \\
(1,073)\end{array}$ & $\begin{array}{c}0.070^{\star * \star} \\
(0,024)\end{array}$ & $\begin{array}{l}-0.038^{\star \star} \\
(0,017)\end{array}$ & $\begin{array}{c}0.063^{* \star *} \\
(0,020)\end{array}$ & $\begin{array}{l}-0,014 \\
(0,014)\end{array}$ & $\begin{array}{c}1.754^{\star \star *} \\
(0,491)\end{array}$ & $\begin{array}{l}-0,719 \\
(0,441)\end{array}$ \\
\hline Grupo & $\begin{array}{c}0,121 \\
(0,081)\end{array}$ & $\begin{array}{c}0,117 \\
(0,081)\end{array}$ & $\begin{array}{l}0.152^{*} \\
(0,087)\end{array}$ & $\begin{array}{c}0,141 \\
(0,087)\end{array}$ & $\begin{array}{l}0.144^{\star *} \\
(0,069)\end{array}$ & $\begin{array}{l}0.134^{*} \\
(0,069)\end{array}$ & $\begin{array}{l}0.106^{*} \\
(0,055)\end{array}$ & $\begin{array}{l}0.133^{\star *} \\
(0,055)\end{array}$ & $\begin{array}{c}0,032 \\
(0,053)\end{array}$ & $\begin{array}{c}0,043 \\
(0,051)\end{array}$ & $\begin{array}{l}0.085^{*} \\
(0,051)\end{array}$ & $\begin{array}{l}0.093^{*} \\
(0,050)\end{array}$ & $\begin{array}{l}0.118^{* *} \\
(0,052)\end{array}$ & $\begin{array}{l}0.114^{* *} \\
(0,050)\end{array}$ & $\begin{array}{c}0,008 \\
(0,029)\end{array}$ & $\begin{array}{c}0,01 \\
(0,027)\end{array}$ & $\begin{array}{c}0,022 \\
(0,071)\end{array}$ & $\begin{array}{c}0,026 \\
(0,069)\end{array}$ \\
\hline Competidores & $\begin{array}{l}-0,012 \\
(0,201)\end{array}$ & $\begin{array}{c}0,034 \\
(0,193)\end{array}$ & $\begin{array}{c}0,433 \\
(0,313)\end{array}$ & $\begin{array}{c}0,382 \\
(0,333)\end{array}$ & $\begin{array}{c}0,07 \\
(0,188)\end{array}$ & $\begin{array}{c}0,025 \\
(0,183)\end{array}$ & $\begin{array}{c}-0,12 \\
(0,076)\end{array}$ & $\begin{array}{c}-0,11 \\
(0,075)\end{array}$ & $\begin{array}{c}0,098 \\
(0,120)\end{array}$ & $\begin{array}{c}0,096 \\
(0,117)\end{array}$ & $\begin{array}{l}-0,027 \\
(0,087)\end{array}$ & $\begin{array}{l}-0,026 \\
(0,088)\end{array}$ & $\begin{array}{l}-0,011 \\
(0,048)\end{array}$ & $\begin{array}{l}-0,012 \\
(0,046)\end{array}$ & $\begin{array}{c}0,038 \\
(0,042)\end{array}$ & $\begin{array}{c}0,037 \\
(0,040)\end{array}$ & $\begin{array}{c}0,061 \\
(0,085)\end{array}$ & $\begin{array}{c}0,068 \\
(0,083)\end{array}$ \\
\hline Clientes & $\begin{array}{c}0,069 \\
(0,085)\end{array}$ & $\begin{array}{c}0,054 \\
(0,084)\end{array}$ & $\begin{array}{c}0,015 \\
(0,088)\end{array}$ & $\begin{array}{l}-0,016 \\
(0,085)\end{array}$ & $\begin{array}{l}0.140^{*} \\
(0,080)\end{array}$ & $\begin{array}{c}0,113 \\
(0,083)\end{array}$ & $\begin{array}{c}0.128^{\star \star *} \\
(0,046)\end{array}$ & $\begin{array}{l}0.118^{\star *} \\
(0,046)\end{array}$ & $\begin{array}{c}0.126^{\star \star \star} \\
(0,046)\end{array}$ & $\begin{array}{l}0.116^{\star *} \\
(0,046)\end{array}$ & $\begin{array}{c}0.172^{\star \star *} \\
(0,044)\end{array}$ & $\begin{array}{c}0.163^{* * *} \\
(0,044)\end{array}$ & $\begin{array}{c}0.117^{\star \star \star} \\
(0,032)\end{array}$ & $\begin{array}{c}0.100^{\star \star \star} \\
(0,031)\end{array}$ & $\begin{array}{c}0.148^{\star \star *} \\
(0,029)\end{array}$ & $\begin{array}{c}0.135^{* \star *} \\
(0,028)\end{array}$ & $\begin{array}{c}0.358^{\star * *} \\
(0,052)\end{array}$ & $\begin{array}{c}0.330^{\star * *} \\
(0,051)\end{array}$ \\
\hline Proveedores & $\begin{array}{c}0.255^{\star \star \star} \\
(0,082)\end{array}$ & $\begin{array}{c}0.243^{\star \star \star} \\
(0,084)\end{array}$ & $\begin{array}{l}0.168^{*} \\
(0,086)\end{array}$ & $\begin{array}{c}0,131 \\
(0,087)\end{array}$ & $\begin{array}{l}0.170^{\star \star} \\
(0,083)\end{array}$ & $\begin{array}{l}0.138^{*} \\
(0,080)\end{array}$ & $\begin{array}{c}0.256^{\star \star \star} \\
(0,040)\end{array}$ & $\begin{array}{c}0.230 \star \star \star \\
(0,041)\end{array}$ & $\begin{array}{l}0.227^{\star \star \star} \\
(0,041)\end{array}$ & $\begin{array}{c}0.202^{\star \star \star} \\
(0,041)\end{array}$ & $\begin{array}{c}0.216^{\star \star \star} \\
(0,043)\end{array}$ & $\begin{array}{c}0.196^{\star \star \star} \\
(0,043)\end{array}$ & $\begin{array}{c}0.333^{\star \star *} \\
(0,028)\end{array}$ & $\begin{array}{c}0.282^{\star \star *} \\
(0,029)\end{array}$ & $\begin{array}{c}0.132^{\star \star *} \\
(0,023)\end{array}$ & $\begin{array}{c}0.095^{* * *} \\
(0,021)\end{array}$ & $\begin{array}{c}0.310^{\star \star \star} \\
(0,043)\end{array}$ & $\begin{array}{c}0.237^{\star \star \star \star} \\
(0,043)\end{array}$ \\
\hline Organizaciones de $1+D$ & $\begin{array}{c}0,02 \\
(0,096)\end{array}$ & $\begin{array}{c}0,028 \\
(0,094)\end{array}$ & $\begin{array}{c}0,122 \\
(0,110)\end{array}$ & $\begin{array}{c}0,12 \\
(0,111)\end{array}$ & $\begin{array}{c}0,076 \\
(0,086)\end{array}$ & $\begin{array}{c}0,077 \\
(0,089)\end{array}$ & $\begin{array}{l}0.086^{*} \\
(0,050)\end{array}$ & $\begin{array}{c}0,078 \\
(0,049)\end{array}$ & $\begin{array}{l}0.125^{\star *} \\
(0,050)\end{array}$ & $\begin{array}{l}0.114^{\star *} \\
(0,050)\end{array}$ & $\begin{array}{c}0,072 \\
(0,046)\end{array}$ & $\begin{array}{c}0,068 \\
(0,046)\end{array}$ & $\begin{array}{l}0.083^{\star *} \\
(0,042)\end{array}$ & $\begin{array}{l}0.074^{\star} \\
(0,039)\end{array}$ & $\begin{array}{c}0,013 \\
(0,028)\end{array}$ & $\begin{array}{c}0,014 \\
(0,026)\end{array}$ & $\begin{array}{c}0,024 \\
(0,069)\end{array}$ & $\begin{array}{c}0,026 \\
(0,065)\end{array}$ \\
\hline
\end{tabular}

Nota: * Significativo al 10 por $100^{* *}$ Significativo al 5 por 100*** Significativo al 1 por 100. Las desviaciones típicas robustas que se encuentra en paréntesis. 


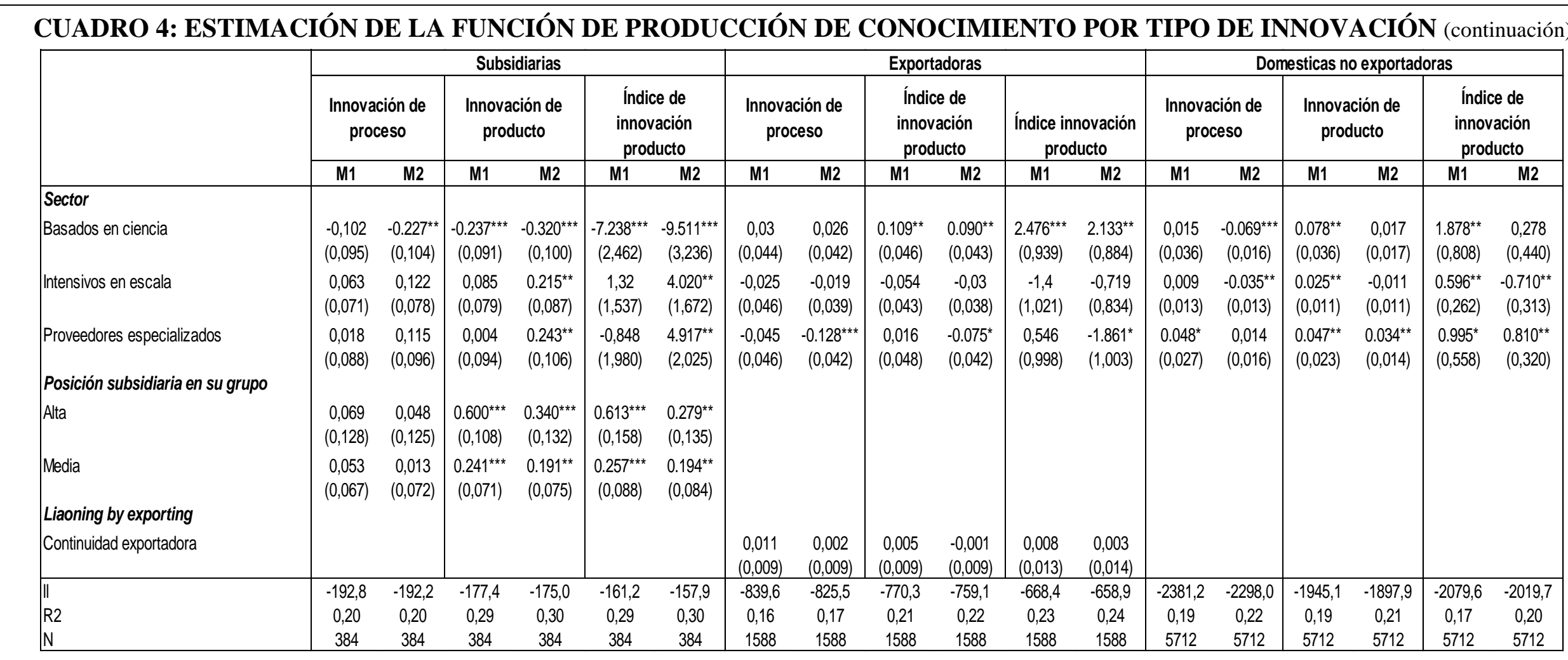

Nota: * Significativo al 10 por $100 * *$ Significativo al 5 por $100 * * *$ Significativo al 1 por 100 . Se registran los efectos marginales (a la media de la muestra) y

las desviaciones típicas robustas que se encuentra en paréntesis. 
Otra variable que impacta positivamente la innovación de producto en las empresas internacionalizadas es la pertenencia a un grupo empresarial, es decir, cooperación con otras empresas del grupo. Por último, la cooperación con universidades y centros de I+D sólo parece tener influencia en las innovaciones de las empresas nacionales exportadoras.

De manera similar a los resultados alcanzados en la etapa anterior del modelo, se observa que las empresas domésticas exportadoras ubicadas en sectores basados en la ciencia son las que muestran una mayor probabilidad de innovar, especialmente en productos. Por su parte, en las subsidiarias extranjeras la probabilidad de lograr innovaciones decrece si se está en un sector con base en el avance de la ciencia y se relaciona positivamente con las subsidiarias que se ubican en sectores intensivos en escala o donde priman los proveedores especializados. La posición de la subsidiaria en el grupo multinacional es un factor importante para explicar la innovación de producto pero no la de proceso en este tipo de empresas. Para el caso de las empresas nacionales exportadoras no existe una relación positiva entre la variable que mide el aprendizaje mediante la exportación y la producción de innovaciones.

\subsection{Productividad multifactorial}

A continuación, se muestran las estimaciones de la productividad multifactorial (cuadro 5). Para cada una de estas medidas de la productividad se estimaron cuatro tipos de modelos. M1A toma como variables independientes la innovación de producto y proceso predichas en la etapa anterior teniendo en cuenta el gasto en I+D. M1B utiliza como regresores la innovación en proceso y el índice de innovación de producto pronosticados, nuevamente utilizando las estimaciones de la etapa anterior donde el gasto en I+D estimado de la primera etapa servía de regresor. De la misma forma, M2A y M2B toman los mismos valores predichos para la innovación pero cambia el tipo de gasto a AI. El resto de variables se mantiene idéntico para los cuatro modelos.

Las estimaciones en el cuadro 5 evidencian que la innovación de producto, conectada con la inversión en $\mathrm{I}+\mathrm{D}$, tiene un efecto estadísticamente significativo y positivo sobre la productividad multifactorial tanto en las subsidiarias como en las empresas nacionales exportadoras. Sin embargo, no existe un impacto significativo de las innovaciones de proceso sobre la productividad. Por su parte, el índice de innovación de producto tan sólo tiene efecto positivo sobre la productividad en el caso de las empresas domésticas exportadoras. Estos resultados sugieren que otras variables pueden ser más importantes para explicar la productividad, entre ellas el capital de las empresas y el sector al que pertenecen, en especial aquellos basados en la ciencia, como lo muestra la magnitud y la significancia de estas variables en el modelo.

En relación al resto de variables incluidas en el modelo, la edad de la empresa en las subsidiarias no tiene impacto sobre la productividad mientras que su posición en la corporación sigue manteniendo su poder explicativo como determinante de su desempeño productivo. En el caso de las domésticas exportadoras existe una relación inversa entre el tiempo de establecimiento y las ganancias en productividad y, además, el aprendizaje mediante la exportación no afecta a la productividad. 


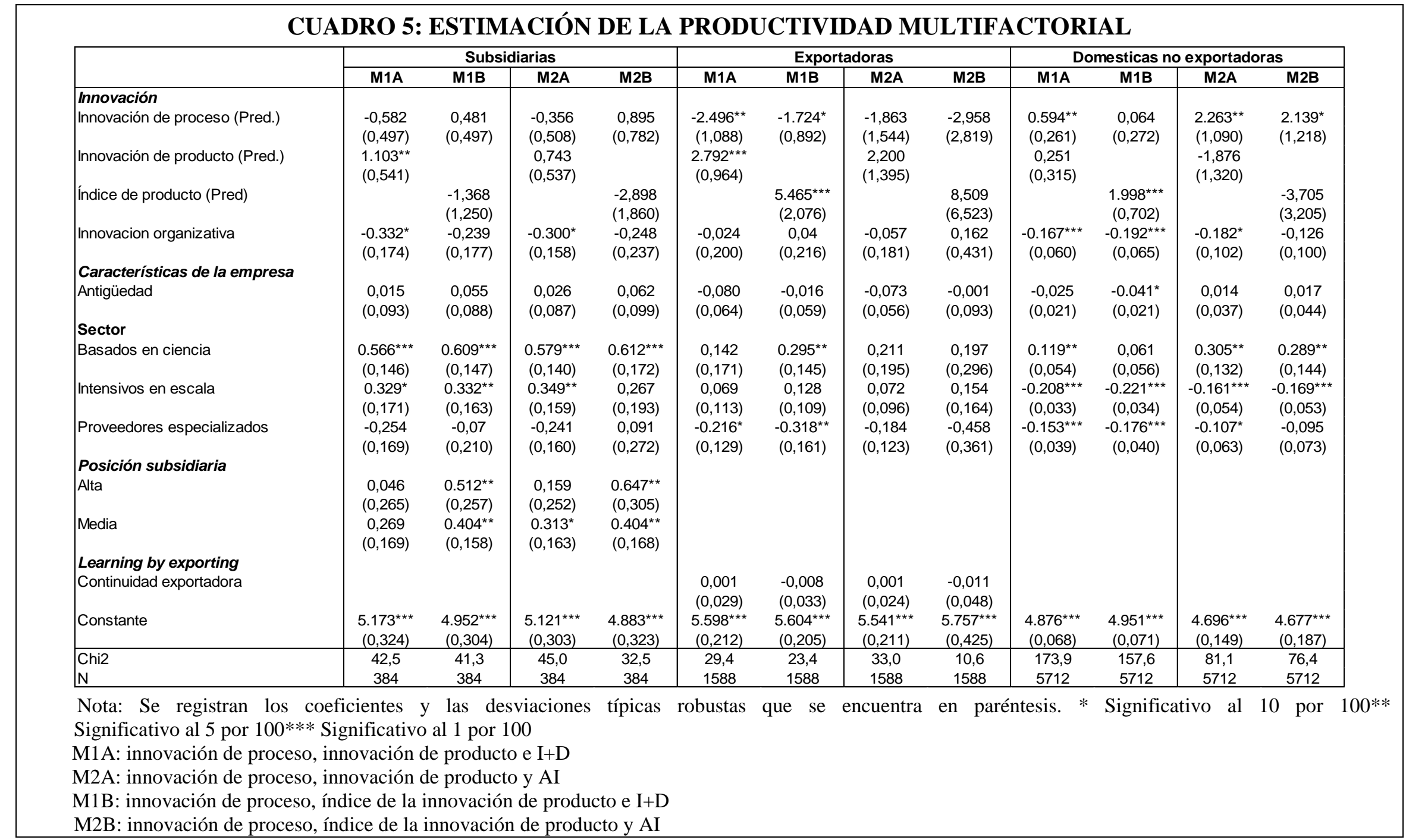




\section{CONCLUSIONES}

En esta investigación se han evaluado los determinantes de la innovación y su efecto sobre la productividad en las empresas extranjeras y las empresas nacionales que exportan. Su propósito consiste en distinguir rasgos diferenciadores de los procesos de innovación y su efecto sobre la productividad de este tipo de empresas frente a las firmas que no están conectadas en los mercados internacionales.

De las estimaciones sobre la decisión e intensidad de invertir tanto en I+D como en actividades de innovación (AI) se desprenden varias conclusiones. Las variables que tienen mayor relevancia para explicar los procesos de innovación en las subsidiarias extranjeras y las empresas domésticas exportadoras son el tamaño empresarial, el personal cualificado, la protección estratégica de la innovación y el pertenecer a sectores basados en la ciencia. El principal obstáculo que afecta a las actividades de innovación es la limitación de recursos tanto para empresas nacionales exportadoras como subsidiarias; pero mientras que la ausencia de personal cualificado es importante para las nacionales que exportan, esta variable no es tan relevante para las subsidiarias para quienes la información de mercado constituye una limitación fundamental. Se encontró evidencia de que la posición de las subsidiarias en el grupo multinacional tiene efectos sobre sus capacidades de innovación. Esto apunta, además, que las subsidiarias más integradas en los mercados externos y con actividades formales de I+D podrían estar desarrollando capacidades de innovación que les permiten acercarse a una estrategia de creación de competencias.

Los resultados obtenidos de la estimación de la función de producción de conocimiento que relaciona los insumos con la producción de innovaciones, apuntan a que, mientras el esfuerzo en I+D y en AI se ve reflejado en una mayor probabilidad de innovar, cualquiera que sea el tipo de empresa analizado, hay diferencia en el nivel de sus efectos. En primer lugar, la I+D parece tener un mayor rendimiento en términos de producción de innovaciones de producto y de proceso en comparación con el gasto total en actividades de innovación. En segundo lugar, mientras que en las empresas domésticas, tanto en las que exportan como en las que no, la inversión en I+D tiene un alto impacto sobre la innovación, en las subsidiarias se observa una elevada dependencia de otras actividades, como puede ser la compra de maquinaria y equipo, en los procesos de producción de innovaciones.

Si bien los subsidios públicos para financiar actividades de innovación son importantes para explicar la probabilidad y el esfuerzo innovador (etapa I del modelo), no se advierten tan relevantes para explicar la producción de innovaciones. Esto indica que es necesario ajustar los mecanismos de política dirigidos a mejorar el rendimiento de la inversión en innovación que se financia públicamente. En cuanto a las condiciones de apropiación del conocimiento, se evidencia que el efecto de la protección formal de la innovación es más apreciable en las subsidiarias extranjeras, mientras que en las domésticas exportadoras son importantes tanto los mecanismos formales como los estratégicos, siendo estos últimos los de mayor impacto.

La cooperación con proveedores, en primer lugar, y con el grupo empresarial, en segundo, tienen los mayores efectos en la obtención de innovaciones en todos los grupos analizados, siendo la cooperación con clientes también de relevancia para las empresas nacionales exportadoras pero no tanto para las subsidiarias extranjeras. Por su parte, la cooperación con universidades y centros de I+D sólo parece tener influencia en los procesos de innovación de las empresas domésticas que exportan.

La posición de la subsidiaria en su grupo multinacional es un factor importante para explicar la innovación en este tipo de empresas. Este resultado refuerza el argumento de que 
la posición que detentan las subsidiarias es relevante para distinguir aquellas unidades extranjeras que podrían estar desarrollando capacidades de innovación y que además podrían ser fuentes de conocimientos que fluyen hacia la economía local.

Finalmente, las innovaciones de proceso no parecen tener efecto alguno sobre la productividad de las empresas internacionalizadas. En contraste, la innovación de producto tienen un efecto significativo y positivo en el desempeño productivo, especialmente cuando se encuentra conectada a la inversión en I+D, pero no necesariamente al resto de la inversión en actividades de innovación. Este resultado indicaría que para maximizar los rendimientos de productividad de la innovación resultan mucho más importantes los esfuerzos internos de las empresas por desarrollar capacidades tecnológicas avanzadas en contraposición a la mera compra de tecnología externa. El índice de innovación de producto tan sólo tiene efecto positivo sobre la productividad en el caso de las empresas domésticas exportadoras.

Hay dos limitaciones centrales en el estudio. La primera es la imposibilidad de identificar a las empresas nacionales que han logrado expandir sus actividades a través de la inversión extranjera, es decir, a las empresas multinacionales domésticas, las cuales en la literatura se ha encontrado que poseen mayores capacidades tecnológicas que el resto de empresas en la economía. La segunda es la utilización de datos de sección cruzada, que a diferencia de las estimaciones con datos de panel, no permiten capturar los efectos dinámicos del modelo y controlar los factores inobservables asociados a las características específicas de las firmas. A futuro es importante incorporar la dinámica en la investigación a través del uso de datos longitudinales, incluir a las multinacionales colombianas como unidad de análisis y profundizar en la caracterización del tipo de estrategias tecnológicas que llevan a cabo las subsidiarias extranjeras y las empresas exportadoras nacionales.

\section{BIBLIOGRAFÍA}

Aitken, B. y Harrison, A. (1999): "Do domestic firms benefit from direct foreign investment? Evidence from Venezuela", The American Economic Review, no 89(3), pp. 605-618.

Almeida, R. y Fernandes, A. (2008): “Openness and technological innovations in developing countries: evidence from firm-level surveys", The Journal of Development Studies, $\mathrm{n}^{\circ}$ 44(5), pp. 701-727.

Álvarez, I. y Cantwell, J. (2011): "International Integration and Mandates of Innovative Subsidiaries in Spain”, International Journal of Institutions and Economies, $\mathrm{n}^{\circ} 3(3)$, pp. 415-444.

Álvarez, I. y Marín, R. (2010): “Entry modes and national systems of innovation”, Journal of International Management, $\mathrm{n}^{\mathrm{o}}$ 16(4), pp. 340-353.

Annique, U. y Montoro, A. (2010): "Public funding for product, process and organisational innovation in service industries", The Service Industries Journal, no 30(1), pp. 133147.

Arrow, K (1962). "Economic welfare and the allocation of resources for invention", The rate and direction of inventive activity: Economic and social factors, Princeton University Press, pp. 609-626.

Aw, B. Y., Roberts, M. J. y Winston, T. (2005): "The complementary role of exports and R\&D investments as sources of productivity growth", $\mathrm{n}^{\circ}$ w11774, National Bureau of Economic Research.

Balcet, G. y Evangelista, R. (2005): "Global technology: innovation strategies of foreign affilliates in Italy", Transnational corporations, $\mathrm{n}^{\circ}$ 14(2), p. 53. 
Bayona, C., Garcia-Marco, T. y Huerta, E. (2001): "Firms' motivations for cooperative R\&D: an empirical analysis of Spanish firms", Research Policy, Elsevier, no 30(8), pp. 12891307.

Belderbos, R., Carree, M., Diederen, B., Lokshin, B. y Veugelers, R. (2004): "Heterogeneity in R\&D cooperation strategies", International Journal of Industrial Organization, $\mathrm{n}^{\circ}$ 22(8), pp. 1237-1263.

Bellak, C. (2004): "How domestic and foreign firms differ and why does it matter? ", Journal of economic surveys, $\mathrm{n}^{\circ} 18(4), 483-514$.

Berger, M. y Diez, J. (2008): "Can host innovation systems in late industrializing countries benefit from the presence of transnational corporations? Insights from Thailand's manufacturing industry", European Planning Studies, no 16(8), 1047-1074.

Birkinshaw, J. y Hood, N. (1998): "Multinational subsidiary evolution: capability and charter change in foreign-owned subsidiary companies", Academy of management review, $\mathrm{n}^{\circ}$ 23(4), pp. 773-795.

Bozeman, B. y Link, A. (1983): Investments in Technology: Corporate Strategy and Public Policy Alternatives, Ed. Praeger, New York.

Bratti, M. y Felice, G. (2012): “Are exporters more likely to introduce product innovations? ”, The World Economy, no 35(11), pp. 1559-1598.

Cameron, A. y Trivedi, P. (2009): Microeconometrics using stata (vol. 5), Ed. Stata Press College Station, TX. New York.

Cantwell, J. y Molero, J. (2003): Multinational enterprises, innovative strategies and systems of innovation", Ed. Edward Elgar Publishing. Northampton.

Cantwell, J. y Mudambi, R. (2005): "MNE competence creating subsidiary mandates", Strategic Management Journal, no 26(12), pp. 1109-1128.

Cantwell, J. y Santangelo, G. D. (2000): "Capitalism, profits and innovation in the new techno-economic paradigm", Journal of Evolutionary Economics, $\mathrm{n}^{\circ} 10(1)$, pp. 131157.

Cassiman, B., Golovko, E., y Martínez-Ros, E. (2010): "Innovation, exports and productivity", International Journal of Industrial Organization, n 28(4), pp. 372-376.

Cassiman, B. y Veugelers, R. (2002): "R\&D cooperation and spillovers: some empirical evidence from Belgium", The American Economic Review, no 92(4), pp. 1169-1184.

Castellani, D. y Zanfei, A. (2007): "Internationalisation, Innovation and Productivity: How Do Firms Differ in Italy?", The World Economy, no 30(1), pp. 156-176.

Clerides, S., Lach, S. y Tybout, J. (1998): "Is learning by exporting important? Microdynamic evidence from Colombia, Mexico, and Morocco", Quarterly Journal of Economics, n $^{\circ}$ 113(3), pp. 903-947.

Cohen, W. M. (2010): "Fifty years of empirical studies of innovative activity and performance", Handbook of the Economics of Innovation, $\mathrm{n}^{\circ}$ 1, pp. 129-213.

Cohen, W. M. y Klepper, S. (1996): "Firm size and the nature of innovation within industries: the case of process and product R\&D", The Review of Economics and Statistics, $\mathrm{n}^{\circ}$ 78, pp. 232-243.

Cohen, W. M. y Levinthal, D. A. (1990): “Absorptive capacity: a new perspective on learning and innovation”, Administrative Science Quarterly, pp. 128-152. 
Crépon, B., Duguet, E., y Mairesse, J. (1998): "Research, Innovation and Productivity: An Econometric Analysis At The Firm Level", Economics of Innovation and New technology, $\mathrm{n}^{\mathrm{o}} 7(2)$, pp. 115-158.

Criscuolo, C., Haskel, J. y Slaughter, M. (2010): "Global engagement and the innovation activities of firms", International Journal of Industrial Organization, $\mathrm{n}^{\circ} 28(2)$, pp. 191-202.

Dachs, B., Ebersberger, B. y Lööf, H. (2008): "The innovative performance of foreign-owned enterprises in small open economies", The Journal of Technology Transfer, $\mathrm{n}^{\circ} 33(4)$, pp. 393-406.

David, P. A., Hall, B. H. y Toole, A. A. (2000): "Is public R\&D a complement or substitute for private R\&D? A review of the econometric evidence", Research Policy, no 29(4), pp. 497-529.

Doms, M., y Jensen, J. (1998): Comparing wages, skills, and productivity between domestically and foreign-owned manufacturing establishments in the United States, Ed. University of Chicago, Chicago.

Dosi, G. (1988): "Sources, procedures, and microeconomic effects of innovation", Journal of Economic Literature, $\mathrm{n}^{\circ}$ 23, pp. 1120-1171.

Dunning, J. (1994): “The Eclectic Paradigm of International Production: A Restatement and Some Possible Extensions”, Journal of International Business Studies, $\mathrm{n}^{\circ} 19$ (1), pp. 131.

Ebersberger, B. y Lööf, H. (2004): "Innovation behaviour and productivity performance in the nordic region does foreign ownership matter?”, CESIS WP n 27, The Royal Institute of Technology Centre of Excellence for Science and Innovation Studies. Stockholm.

Freel, M. S. (2005): "Patterns of innovation and skills in small firms", Technovation, $\mathrm{n}^{\circ}$ 25(2), pp. 123-134.

Frenz, M. y Ietto-Gillies, G. (2007): "Does multinationality affect the propensity to innovate? An analysis of the third UK Community Innovation Survey", International Review of Applied Economics, $\mathrm{n}^{\circ}$ 21(1), pp. 99-117.

Girma, S., Kneller, R. y Pisu, M. (2005): "Exports versus FDI: an empirical test". Review of World Economics, $\mathrm{n}^{\circ}$ 141(2), pp. 193-218.

Greene, W. (1999): Econometric Analysis (7th Edition), Ed. Prentice Hall, New Jersey.

Griffith, R., Huergo, E., Mairesse, J. y Peters, B. (2006): "Innovation and productivity across four European countries", Oxford Review of Economic Policy, n 22(4), pp. 483-498.

Griffith, R., Redding, S. y H., S. (2002): "Productivity convergence and foreign ownership at the establishment level", The Institute for Fiscal Studies Working Paper $n^{0}$ 02/22, Centre for Economic Performance, London School of Economics, London

Griliches, Z. (1979): "Issues in assessing the contribution of research and development to productivity growth", Bell Journal of Economics, $\mathrm{n}^{\circ}$ 10, pp. 92-116.

Heckman, J. (1979): "Sample Selection Bias as a Specification Error", Econometrica, no 47, $\mathrm{n}^{\circ} .1$ (January), pp. 153-161.

Helpman, E., Melitz, M. y Yeaple, E. (2004): "Export Versus FDI with Heterogeneous Firms", The American Economic Review, no 94 (1).

Hitt, M., Hoskisson R. y Kim H. (1997): "International diversification: Effects on innovation and firm performance in product-diversified firms", Academy of Management journal, $n^{\circ} 40(4)$, pp. 767-798. 
Hurmerinta-Peltomäki, L. (1996): "The innovation and internationalisation of small businesses: Applying the innovation concept in an export context", Letter from the editor, $\mathrm{n}^{\mathrm{o}} 43$.

Jaramillo, H., Lugones, G., y Salazar, M. (2001): Manual de Bogotá. Ed. OEA/ RICYT/ COLCIENCIAS/ CYTED/ OCyT. Bogotá.

Jefferson, G., Huamao, B., Xiaojing, G. y Xiaoyun, Y. (2006): "R\&D performance in Chinese industry", Economics of Innovation and New technology, no 15(4-5), pp. 345-366.

Klette, T. J. y Kortum, S. (2002): "Innovating firms and aggregate innovation", Journal of Political Economy, n ${ }^{\circ}$ 112(5), pp.986-1018.

Levinsohn, J. y Petrin, A. (2003): "Estimating production functions using inputs to control for unobservables", The Review of Economic Studies, 70(2), 317-341.

Malerba, F. (2002): "Sectoral systems of innovation and production", Research Policy, no $31(2), 247-264$.

Marin, A. y Bell, M. (2010): “The local/global integration of MNC subsidiaries and their technological behaviour: Argentina in the late 1990s", Research Policy, n ${ }^{\circ}$ 39(7), pp. 919-931.

Nelson, R. R. y Winter, S. G. (1982): An Evolutionary Theory of Economic Change. Massachusetts and London, Ed. Cambridge and Belknap Press. Cambridge.

Pavitt, K. (1984) "Sectoral patterns of technical change: towards a taxonomy and a theory", Research Policy, $\mathrm{n}^{\circ}$ 13(6), pp. 343-373.

Salomon, R. M. y Shaver, J. M. (2005): "Learning by exporting: new insights from examining firm innovation", Journal of Economics and Management Strategy, $\mathrm{n}^{\circ}$ 14(2), pp. 431460.

Siedschlag, I., Zhang, X. y Cahill, B. (2010): “The effects of the internationalization of firms on innovation and productivity", ESRI working paper $\mathrm{n}^{\circ} 363$, Economic and Social Research Institute, Dublin

Tether, B. S. (2002): "Who co-operates for innovation, and why: An empirical analysis, Research policy", n 31(6), pp. 947-967.

Tidd, J. y Pavitt, K. (2005): Managing innovation: integrating technological, market and organizational change", Ed. John Wiley and Sons, Ltda. Chichester.

Wagner, J. (2006): "Exports, foreign direct investment, and productivity: Evidence from German firm level data", Applied Economics Letters, $\mathrm{n}^{\circ}$ 13(6), pp. 347-349. 
Anexo 1. Definición de variables y estadísticos descriptivos

\begin{tabular}{|c|c|c|c|c|c|}
\hline & Variable & Definición & Observaciones & Media & $\sigma$ \\
\hline Inversión & $\begin{array}{l}\text { Intensidad I+D (Log) } \\
\text { Intensidad ACTI (Log) } \\
\text { Probabilidad I+D } \\
\text { Probabilidad ACTI } \\
\end{array}$ & $\begin{array}{l}\text { Logaritmo de la inversión en I+D por trabajador. } \\
\text { Logaritmo de la inversión en actividades de innovación por trabajador. } \\
\text { Dummy igual a } 1 \text { si la empresa ha realizado inversiones en I+D. } \\
\text { Dummy igual a } 1 \text { si la empresa ha invertido en actividades de innovación (AI). }\end{array}$ & $\begin{array}{l}832 \\
2969 \\
7824 \\
7824 \\
\end{array}$ & $\begin{array}{l}2,396 \\
3,390 \\
0,110 \\
0,380 \\
\end{array}$ & $\begin{array}{l}0,235 \\
0,211 \\
0,310 \\
0,490 \\
\end{array}$ \\
\hline Innovación & $\begin{array}{l}\text { Innovación de proceso } \\
\text { Innovación de producto } \\
\text { Índice de innovación producto }\end{array}$ & $\begin{array}{l}\text { Dummy igual a } 1 \text { si la empresa obtuvo bienes o servicios nuevos o significativamente mejorados para el } \\
\text { mercado internacional. } \\
\text { Dummy igual a } 1 \text { si la empresa obtuvo bienes o servicios nuevos o significativamente mejorados para el } \\
\text { mercado internacional. } \\
\text { Mide la novedad de las innovaciones de producto introducidas. Se pondera la novedad de la innovación de } \\
\text { acuerdo a la diferencia entre el número de innovaciones obtenidas (empresa, nacional o internacional) sobre el } \\
\text { total de innovaciones que obtienen las empresas nacionales. }\end{array}$ & 7824 & $\begin{array}{l}0,238 \\
0,184 \\
0,09\end{array}$ & $\begin{array}{l}0,426 \\
0,388 \\
0,21\end{array}$ \\
\hline Productividad & Productividad multifactorial & Productividad multifactorial calculada mediante el método de Levinsohn y Petrin (2003). & 7824 & 4,991 & 0,995 \\
\hline Recurso humano & $\begin{array}{l}\text { Personal cualificado } \\
\text { Personal altamente cualificado }\end{array}$ & $\begin{array}{l}\text { Porcentaje del personal empleado con educación técnica, tecnóloga y profesional. } \\
\text { Porcentaje del personal empleado con educación de maestría y doctorado. }\end{array}$ & $\begin{array}{l}7824 \\
7824\end{array}$ & $\begin{array}{l}0,280 \\
0,003\end{array}$ & $\begin{array}{l}0,240 \\
0,016\end{array}$ \\
\hline $\begin{array}{l}\text { Características } \\
\text { empresa }\end{array}$ & $\begin{array}{l}\text { Antigüedad } \\
\text { Cuota de mercado } \\
\text { Tamaño }\end{array}$ & $\begin{array}{l}\text { Logaritmo del personal en años. } \\
\text { Ventas de la empresa sobre las ventas del sector al que pertenece a CIIU a tres dígitos. } \\
\text { Logaritmo del personal empleado. }\end{array}$ & $\begin{array}{l}7824 \\
7824 \\
7824 \\
\end{array}$ & $\begin{array}{l}2,853 \\
0,001 \\
7,427\end{array}$ & $\begin{array}{l}0,738 \\
0,006 \\
2,361\end{array}$ \\
\hline Apoyo público & Financiación pública ACTI & Dummy igual a 1 si la empresa recibió recursos públicos para desarrollar AI. & 7824 & 0,001 & 0,994 \\
\hline $\begin{array}{l}\text { Apropiación del } \\
\text { conocimiento }\end{array}$ & $\begin{array}{l}\text { Protección formal } \\
\text { Protección estratégica }\end{array}$ & $\begin{array}{l}\text { Dummy igual a } 1 \text { si la empresa utilizó patentes de invención, modelos de utilidad, derechos de autor, registros } \\
\text { de software, registro de diseños industriales, registro de signos distintivos y marcas y certificados de obtención } \\
\text { de variedades vegetales para proteger sus invenciones o innovaciones. } \\
\text { Dummy igual a } 1 \text { si la empresa utilizó para proteger sus invenciones o innovaciones mecanismos como el } \\
\text { secreto industrial, alta complejidad en el diseño, acuerdos o contratos de confidencialidad con otras empresas y } \\
\text { acuerdos o contratos de confidencialidad con los empleados. }\end{array}$ & 7824 & 0,272 & 0,445 \\
\hline $\begin{array}{l}\text { Fuentes de ideas } \\
\text { para innovar }\end{array}$ & $\begin{array}{l}\text { Grupo } \\
\text { Clientes } \\
\text { Proveedores } \\
\text { Competidores } \\
\text { Universidades/centros de I+D }\end{array}$ & $\begin{array}{l}\text { Dummy igual a } 1 \text { si la empresa utilizó como fuentes de información para la innovación el grupo al que } \\
\text { pertenece. } \\
\text { Dummy igual a } 1 \text { si la empresa utilizó como fuentes de información para la innovación a los clientes. } \\
\text { Dummy igual a } 1 \text { si la empresa utilizó como fuentes de información para la innovación a los proveedores. } \\
\text { Dummy igual a } 1 \text { si la empresa utilizó como fuentes de información para la innovación a sus competidores. } \\
\begin{array}{l}\text { Dummy igual a } 1 \text { si la empresa utilizó como fuentes de información para la innovación a las universidades y } \\
\text { centros de I+D (Centros de Desarrollo Tecnológico -CDT y Centros de investigación). }\end{array}\end{array}$ & $\begin{array}{l}7824 \\
7824 \\
7824 \\
7824\end{array}$ & $\begin{array}{l}0,048 \\
0,218 \\
0,178 \\
0,118\end{array}$ & $\begin{array}{l}0,213 \\
0,413 \\
0,383 \\
0,323\end{array}$ \\
\hline
\end{tabular}




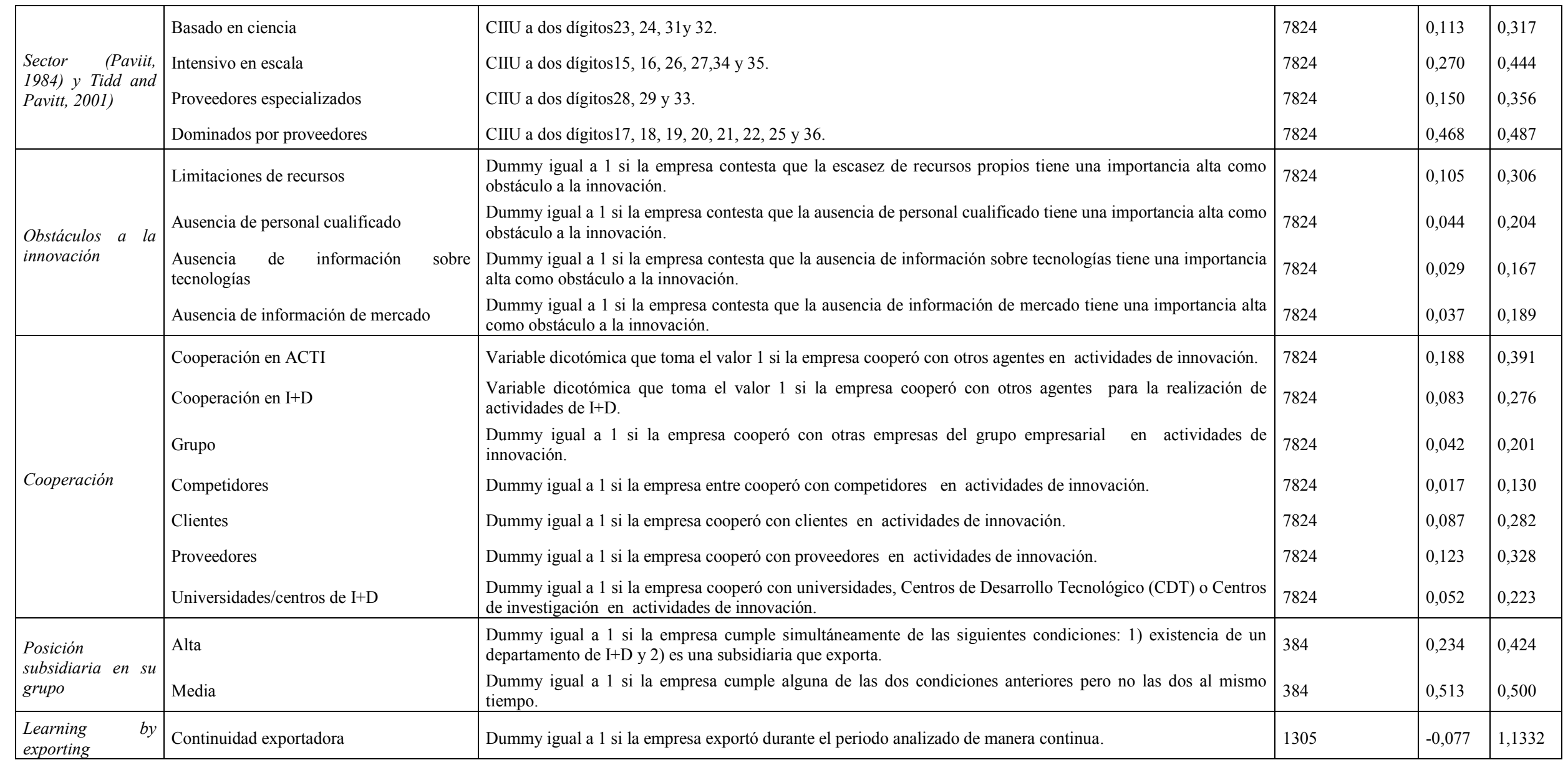

